\title{
The C-terminal helix of BubR1 is essential for CENP-E-dependent chromosome alignment
}

\author{
Thibault Legal $^{1}$, Daniel Hayward ${ }^{2}$, Agata Gluszek-Kustusz $^{1}$, Elizabeth A. Blackburn ${ }^{1}$, Christos Spanos $^{1}$, \\ Juri Rappsilber ${ }^{1,3}$, Ulrike Gruneberg ${ }^{2}$ and Julie P. I. Welburn ${ }^{1, *}$
}

\begin{abstract}
During cell division, misaligned chromosomes are captured and aligned by motors before their segregation. The CENP-E motor is recruited to polar unattached kinetochores to facilitate chromosome alignment. The spindle checkpoint protein BubR1 (also known as BUB1B) has been reported as a CENP-E interacting partner, but the extent to which BubR1 contributes to CENP-E localization at kinetochores has remained controversial. Here we define the molecular determinants that specify the interaction between BubR1 and CENP-E. The basic C-terminal helix of BubR1 is necessary but not sufficient for CENP-E interaction, and a minimal key acidic patch on the kinetochore-targeting domain of CENP-E is also essential. We then demonstrate that BubR1 is required for the recruitment of CENP$E$ to kinetochores to facilitate chromosome alignment. This BubR1CENP-E axis is critical for alignment of chromosomes that have failed to congress through other pathways and recapitulates the major known function of CENP-E. Overall, our studies define the molecular basis and the function for CENP-E recruitment to BubR1 at kinetochores during mammalian mitosis.
\end{abstract}

This article has an associated First Person interview with the first author of the paper.

KEY WORDS: CENP-E, Motor, Kinetochore, Mitosis, Microtubule

\section{INTRODUCTION}

To maintain their genomic integrity, eukaryotic cells must distribute their DNA equally to the daughter cells. Spindle microtubules mediate the segregation of chromosomes by associating with the kinetochore, a large protein complex that mediates the end-on attachment of chromosomes to microtubules. At mitotic onset, chromosomes are dispersed throughout the cytoplasm, posing a challenge for their capture by microtubules from opposite poles, a prerequisite for their accurate segregation. Multiple pathways involving microtubules and motors co-exist to ensure

\footnotetext{
${ }^{1}$ Wellcome Trust Centre for Cell Biology, School of Biological Sciences, University of Edinburgh, Edinburgh EH9 3BF, Scotland, UK. ${ }^{2}$ Sir William Dunn School of Pathology, University of Oxford, South Parks Road, Oxford OX1 3RE, UK. ${ }^{3}$ Chai of Bioanalytics, Institute of Biotechnology, Technische Universität Berlin, Berlin 10623, Germany.

*Author for correspondence (Julie.Welburn@ed.ac.uk)
}

(D) E.A.B., 0000-0003-1325-9575; J.R., 0000-0001-5999-1310; U.G., 0000-00018344-0500; J.P.I.W., 0000-0002-5440-6060

This is an Open Access article distributed under the terms of the Creative Commons Attribution License (https://creativecommons.org/licenses/by/4.0), which permits unrestricted use,

distribution and reproduction in any medium provided that the original work is properly attributed.

Handling Editor: David Glover

Received 9 March 2020; Accepted 6 July 2020 chromosome congression and bi-orientation (Maiato et al., 2017). A subset of chromosomes that lie outside of the interpolar region during spindle assembly are dependent on CENP-E for congression. CENP-E is a large $312 \mathrm{kDa}$ plus-end-directed kinesin that is recruited to unattached and unaligned kinetochores, and to the outer corona, which expands around kinetochores to maximize microtubule capture (Cooke et al., 1997; Yao et al., 1997; Sacristan et al., 2018). Kinetochore-bound CENP-E moves laterally attached chromosomes to the cell equator along microtubules (Wood et al., 1997; Kapoor et al., 2006). CENP-E may also help sort kinetochorenucleated microtubules and promote end-on attachments and biorientation (Shrestha and Draviam, 2013; Sikirzhytski et al., 2018). CENP-E then remains at aligned kinetochores, albeit with lower levels, where it plays a role in maintaining a robust connection between kinetochores and microtubules during metaphase, and during anaphase as the kinetochores are pulled to opposite poles by depolymerizing microtubules (Brown et al., 1996; Vitre et al., 2014).

CENP-E is enriched at unattached and misaligned kinetochores in early mitosis (reviewed in Craske and Welburn, 2020) and is also found at spindle poles (Maffini et al., 2009). The human CENP-E kinetochore-targeting domain has previously been mapped (Chan et al., 1998). Over the years, CENP-E has been reported to interact with multiple kinetochore proteins: BubR1 (also known as BUB1B), CENP-F, Clasp2, Mad1 (MAD1L1), and other interactors such as Septin, CKAP5, NPM1 (Akera et al., 2015; Chan et al., 1998; Maffini et al., 2009; Zhu et al., 2008; Maliga et al., 2013). Post-translational modifications may also enhance CENP-E targeting to kinetochores (Ashar et al., 2000; Zhang et al., 2008). Overall, the molecular basis for CENP-E recruitment to kinetochores remains poorly understood. Kinetochore recruitment of CENP-E has been previously shown to be dependent on the spindle-checkpoint proteins, budding uninhibited by benzimidazole 1 (Bub1) and Bub1-related (BubR1) mitotic checkpoint Ser/Thr kinases; in Xenopus and DLD-1 cells, CENP-E kinetochore levels are strongly reduced upon BubR1 depletion (Johnson et al., 2004; Mao et al., 2003). Other studies, however, argue CENP-E levels are not affected by BubR1 depletion (Chan et al., 1999; Ciossani et al., 2018; Kops et al., 2004). CENP-E remains at bi-oriented kinetochores after removal of checkpoint proteins, disassembly of the outer corona and throughout anaphase, indicating CENP-E has multiple as-yetunidentified binding partners at the kinetochore (Brown et al., 1996; Cooke et al., 1997; Gudimchuk et al., 2013). Here we characterized the kinetochore targeting domain of CENP-E biophysically and used a non-biased approach to find mitotic partners of CENP-E. We found BubR1 as a major interactor and defined the molecular requirements for the BubR1-CENP-E interaction. Overall, we demonstrate BubR1 contributes to CENPE localization to kinetochores and that this axis is essential for facilitating chromosome alignment. 


\section{RESULTS}

To define the regulation of CENP-E targeting to kinetochores, we quantitatively examined endogenous CENP-E levels at kinetochores during distinct stages of cell division, CENP-E levels were maximal during prometaphase and decreased during metaphase (Fig. S1A,B). Indeed, CENP-E levels were largely comparable to those in prometaphase upon nocodazole-induced depolymerization of microtubules, creating unattached kinetochores (Fig. S1A,B). To analyse the molecular requirements for CENP-E localization, we precisely mapped the regions of CENP-E isoform 1 that target to kinetochores and centrosomes using transient transfection of CENP-E constructs fused to GFP (Fig. 1A). CENP-E $2055-2608$ in the $\mathrm{C}$ terminus of CENP-E, largely similar to the previously published kinetochore-targeting construct 19582628, was necessary and sufficient for targeting to kinetochores in HeLa cells (Fig. 1B,C) (Chan et al., 1998). The shorter CENP$E_{2055-2450}$ construct still showed kinetochore localization, whereas an even shorter CENP-E $2055-2356$ construct targeted weakly to a subset of kinetochores (Fig. 1B,C). This heterogeneous targeting was previously observed and is likely to reflect different attachment states or kinetochore heterogeneity (Chan et al., 1998). In the absence of the first 35 amino acids in this domain, CENP-E $2090-2450$ lost the ability to target to kinetochores (Fig. 1B,C). However, CENP-E $2260-2608$ localized specifically to a region between the two centrioles or associated closely with one centriole both in interphase and mitosis (Fig. S1C). The intercentriole-interacting proteins remain unknown, and this interaction was not pursued further here.

We then tested whether CENP-E $2055-2608$ dimerizes with endogenous CENP-E at kinetochores. We depleted CENP-E using a Cas9 inducible cell line expressing a CENP-E sgRNA (McKinley and Cheeseman, 2017). CENP-E was largely depleted after $72 \mathrm{~h}$ (Fig. 1D,E; +doxycyline). Cells clearly depleted for endogenous CENP-E, as identified by immunofluorescence, were analysed. In the absence of endogenous CENP-E, GFP-CENP-E $2055-2608$ was only weakly targeted to most kinetochores, indicating that CENP$E_{2055-2608}$ recruitment to kinetochores might depend on the fulllength endogenous CENP-E, or that CENP-E removal affects other kinetochore proteins necessary for its recruitment (Fig. 1D; Fig. S1D). However, we observed GFP-CENP-E $2055-2608$ at kinetochores close to spindle poles, suggesting that GFP-CENP$E_{2055-2608}$ was recruited to these kinetochore subpopulations independently of endogenous CENP-E, through another binding partner (Fig. S1D,E). GFP-CENP-E $2055-2608$ appeared to compete with CENP-E at kinetochores, causing a reduction in endogenous CENP-E at kinetochores (Fig. 1E) in agreement with Schaar et al. (1997). Additionally, CENP-E $2055-2608$ transfection caused many chromosomes to become misaligned (Fig. 1D,F,G), presumably by replacing endogenous motor-domain-containing CENP-E at kinetochores. Overall, these results indicate that CENP-E $2055-2608$ targeting to kinetochores competes endogenous CENP-E.

To define the molecular basis for the CENP-E kinetochoretargeting domain, we expressed and purified recombinant CENP-

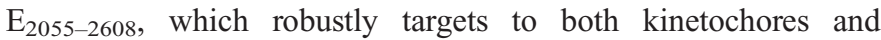
centrosomes, and CENP-E 2055-2358, which targets weakly to kinetochores. CENP-E $2055-2608$ aggregated in $150 \mathrm{mM} \mathrm{NaCl}$ and was maintained in $500 \mathrm{mM} \mathrm{NaCl}$. Size exclusion chromatographymultiangle light scattering (SEC-MALS) analysis revealed that CENP-E $2055-2608$ assembles as a dimer in solution, whereas the minimal kinetochore-targeting domain CENP-E $2055-2358$ was monomeric (Fig. 2A,B). Circular dichroism further defined the secondary structural elements of CENP-E $2055-2608$ and CENP$\mathrm{E}_{2055-2358}$ (Fig. 2C). CENP-E $2055-2608$ has an $\alpha$-helical content of $\sim 50.9 \%$, whereas the shorter domain CENP-E $2055-2358$ is $80 \%$ $\alpha$-helical, with $8.8 \%$ containing turns and $12.1 \%$ containing unstructured regions (Fig. 2D). Rotary shadowing further revealed that CENP-E $2055-2608$ is an elongated domain with a globular region at one end and rod-like shape, supporting a coiled-coil conformation (Fig. 2E). Overall, these data indicated that the CENP-E $2055-2608$ domain is subdivided into an N-terminal monomeric $\alpha$-helical-rich domain, essential for kinetochore targeting, and a C-terminal domain that provides dimerization properties.

We then sought to define the major CENP-E interactors at kinetochores. CENP-E is strongly recruited to unattached kinetochores at mitotic onset (Yen et al., 1991). We incubated CENP-E $2055-2358$ with clarified mitotic cell lysate, from cells arrested using the microtubule-depolymerizing drug nocodazole. We then pulled down CENP-E $2055-2358$ bound to $\mathrm{Ni}^{2+}$-NTA beads and associated proteins, which were subjected to mass spectrometry for identification (Fig. 3A; Table S1). We found that CENP$\mathrm{E}_{2055-2358}$ co-purified with BubR1 and MYPT1, a protein phosphatase 1 regulatory subunit 12A (Fig. 3B). To test whether CENP-E directly binds to BubR1, we expressed and purified recombinant BubR1 from insect cells and analysed whether stoichiometric amounts of BubR1 could interact with the longer dimeric kinetochore-targeting domain CENP-E $2055-2608$, using sizeexclusion chromatography (SEC). Indeed, full-length BubR1-Bub3 complex interacts with CENP-E $2055-2608$ in vitro (data not shown) (Ciossani et al., 2018). We generated two BubR1 constructs containing either the $\mathrm{N}$ - or $\mathrm{C}$-terminal domains. The $\mathrm{N}$ terminus of BubR $_{1-484}$ did not co-migrate with CENP-E $2055-2608$ by SEC (Fig. 3C) whereas the $\mathrm{C}$ terminus containing the pseudokinase domain, BubR1 $1_{432-1050}$, did (Fig. 3D), indicating CENP-E $2055-2608$ binds to the pseudokinase domain of BubR1. While we were conducting these experiments, a parallel study also reported an interaction between CENP-E and the pseudokinase domain of BubR1 $1_{705-1050}$ (Ciossani et al., 2018).

Our in vivo work indicated that CENP- $\mathrm{E}_{2055-2356}$ fused to GFP could still weakly associate with kinetochores (Fig. 1B). To test whether post-translational modifications were necessary for this interaction, we tested whether our recombinant construct CENP$\mathrm{E}_{2055-2358}$ interacted with bacterially expressed BubR $1_{705-1050}$ in vitro. Indeed, CENP-E $2055-2358$ co-eluted with BubR1 $1_{705-1050}$, indicating they interact in the absence of post-translational modifications (Fig. S2A). CENP-E $2055-2358$ is monomeric (Fig. 2A,B) and not very stable in low salt concentrations. To stabilize CENP-E $2055-2608$ while mimicking its dimerization, we fused it to a C-terminal GST tag and removed 14 residues at the $\mathrm{N}$ terminus. CENP-E $2069-2358-\mathrm{GST}$ was more stable in low salt concentration and could then be further used to analyse the BubR1CENP-E interaction by SEC. CENP-E $2069-2358-$ GST co-eluted with BubR $1_{705-1050}$, as shown by the shift in the elution profile (Fig. 4A), while GST alone did not (Fig. S2B). The constructs were monodisperse, but we were not able to obtain diffracting crystals of the kinetochore-targeting domain of CENP-E $2055-2358$ alone or bound to BubR1.

To investigate the thermodynamics of the BubR1-CENP-E interaction, we performed isothermal titration calorimetry (ITC). The CENP-E $2069-2358$-GST construct had to be optimized slightly to remove some GST contaminants and degradation. We therefore removed a further 22 residues at the $\mathrm{N}$ terminus and purified it in complex with BubR1 before separating the complex in high ionic strength using gel filtration. In this way, we obtained $>95 \%$ pure BubR $1_{705-1050}$ and $\mathrm{CENP}_{2091-2358}-\mathrm{GST}$. At $37^{\circ} \mathrm{C}$ the pseudokinase domain of BubR1 bound CENP-E $2091-2358^{-G S T}$ 

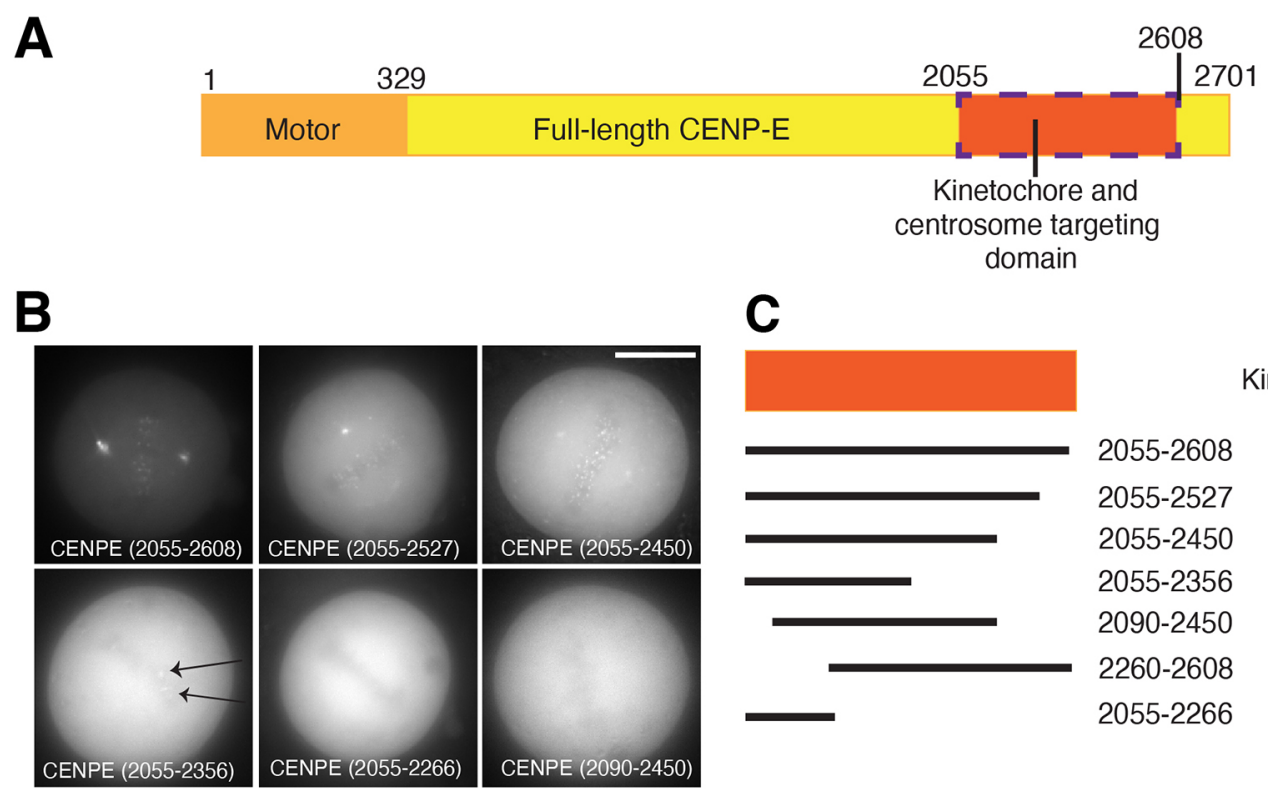

Kinetochore Centrosome

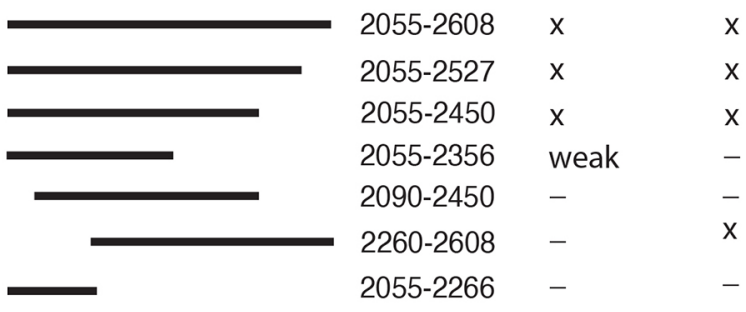

D
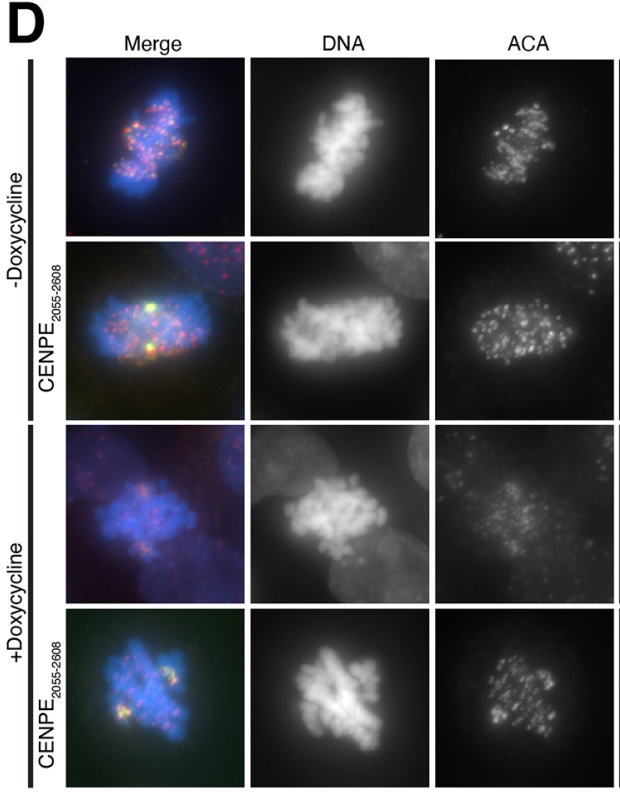

CENP-E

GFP

E
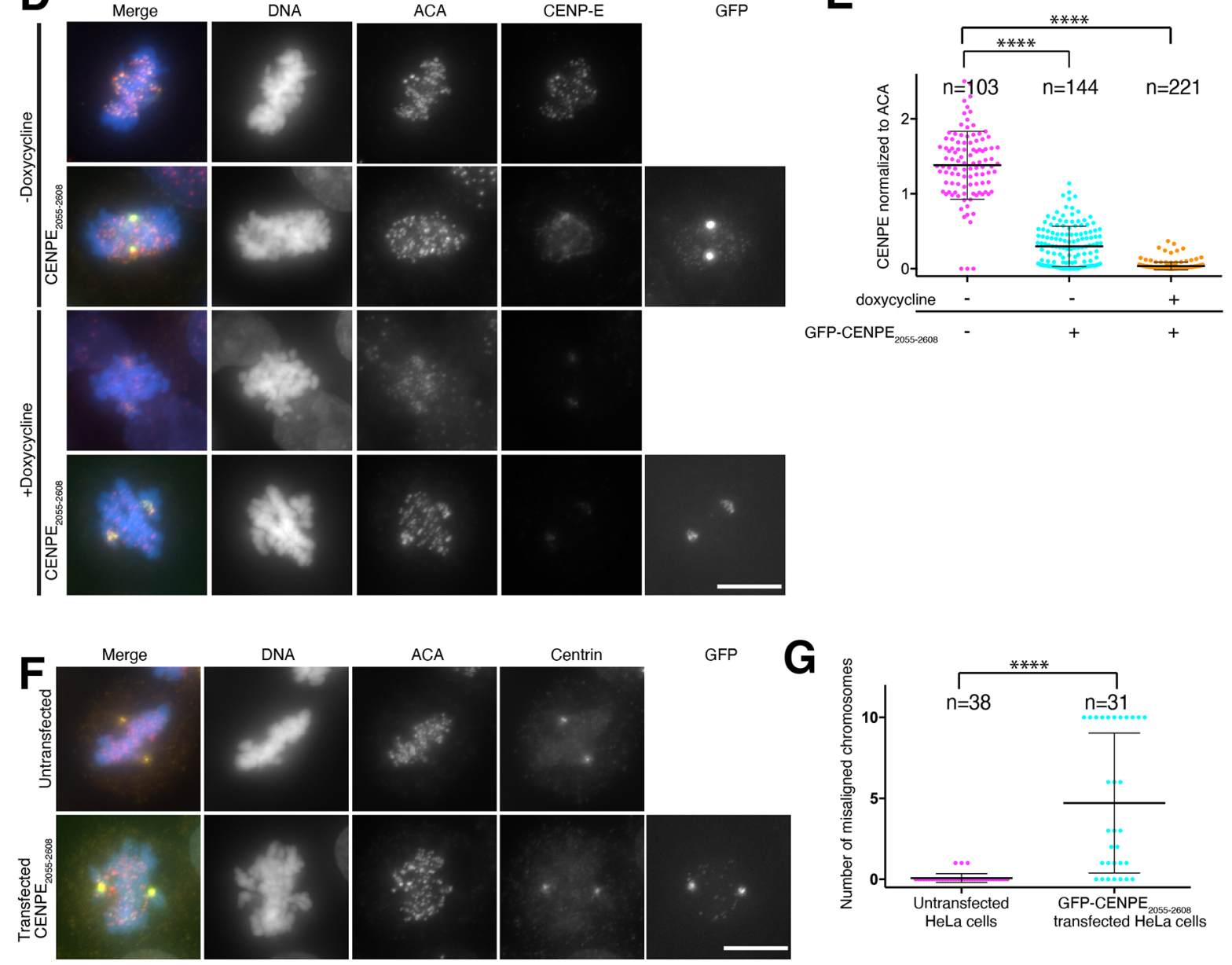

Centrin
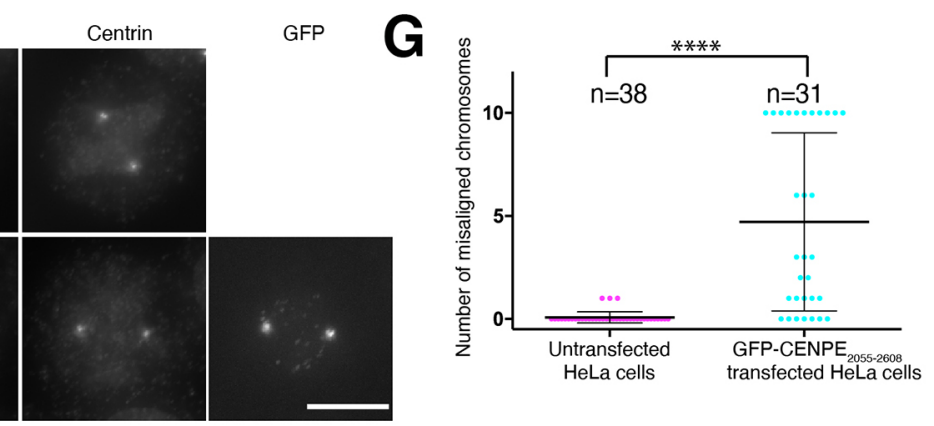

Fig. 1. See next page for legend.

with mid-nanomolar affinity with a $K_{\mathrm{d}}=318 \pm 90 \mathrm{nM}$ (mean \pm s.e.; Fig. 4B). At this temperature, formation of the complex had an exothermic heat signature. The enthalpic and entropic components driving the interaction were of a similar magnitude $(\Delta H=-5.1 \pm 0.2$ $\mathrm{kcal} / \mathrm{mol}$, mean \pm s.e.; $-T \Delta S=-4.1 \mathrm{kcal} / \mathrm{mol})$. The stoichiometry between BubR1 and CENP-E $2091-2358$ was determined to be 1:1 
Fig. 1. Mapping of the kinetochore- and centrosome-targeting domain of CENP-E. (A) Schematic diagram of CENP-E, highlighting the motor and kinetochore- and centrosome-targeting domains. Amino acid residue numbers are indicated. (B) Representative images of live HeLa cells transfected with GFP-CENP-E constructs (arrows indicate kinetochore localization of the construct) and ( $\mathrm{C}$ ) map of the corresponding kinetochore- and centrosometargeting domains for human CENP-E ( $\times$ marks the localization of the construct to the kinetochore or centrosome, - marks the absence of localization). Representative images for CENP- $E_{2260-2608}$ are shown in Fig S1C. (D) Representative immunofluorescence images of HeLa cells transfected with GFP-CENP-E $2055-2608$ in the presence (-Doxycycline) and knockout (+Doxycycline) of endogenous CENP-E and stained for endogenous CENP-E, ACA signal and DNA. (E) Scatter plot showing quantification of CENP-E intensity normalized to ACA signal, in the presence and knockout of endogenous CENP-E (doxycycline - and doxycycline +, respectively) and GFP-CENP-E $2055-2608$. Each point represents the intensity of CENP-E over ACA at one kinetochore, with mean \pm s.d shown. ${ }^{* * *} P<0.0001$ (ordinary oneway ANOVA). (F) Representative immunofluorescence images of wild-type HeLa cells (top) and HeLa cells transfected with GFP-CENP-E $2055-2608$ (bottom) stained for centrin, ACA and DNA. (G) Scatter plot showing the number of misaligned chromosomes in HeLa cells and GFP-CENP$E_{2055-2608}$-transfected HeLa cells. Each point represents one cell with the corresponding number of misaligned chromosomes, with mean \pm s.d shown. ${ }^{* * * *} P<0.0001$ (unpaired $t$-test). Scale bars: $10 \mu \mathrm{m}$.

( $N=0.908 \pm 0.016$, mean \pm s.e.). The stoichiometry must be put in the context of full-length dimeric CENP-E. Thus the CENP-E motor is able to bind to two molecules of BubR1.

We then examined the sequence conservation between Bub1 and BubR1 kinase domains. Previous work highlighted that the $\mathrm{C}$ terminus of Bubl recruits CENP-F to kinetochores (Raaijmakers et al., 2018). We found a predicted helix in the $\mathrm{C}$ terminus of BubR1 that showed sequence divergence between human Bub1 and BubR1, but displayed sequence similarity across BubR1 orthologues in vertebrate species (Fig. 4C). We hypothesized this region might be important for the CENP-E-BubR1 interaction Indeed, CENP-E $2069-2358-$ GST did not co-elute with BubR $1_{705-1030}$ lacking the last 20 amino acids (Fig. 4D) suggesting that this part of BubR1 is critical for the interaction with CENP-E. However, on its own this basic helix in BubR1 $1_{1031-1050}(\mathrm{pI}=10.30)$ fused to MBP, was not sufficient to interact with CENP-E (Fig. 4E), although we cannot rule out that the MBP would disrupt the interaction. Based on the basic properties of this helix, we also mapped the interaction of BubR1 with CENP-E to the C terminus of CENP-E $2055-2358$. We found a negatively charged region in CENP-E, which we hypothesized could interact with the basic helix of the kinase domain of BubR1. We mutated four highly conserved glutamates (E2313, E2316, E2318 and E2319) to alanines in CENP-E $2069-2358$ GST, named thereafter CENP-E ${ }_{4 \mathrm{E}}-\mathrm{GST}$ (Fig. 5A). CENP-E $4 \mathrm{E}^{-} \mathrm{GST}$ was co-incubated with BubR1 $1_{705-1050}$ and analysed by SEC. CENP$\mathrm{E}_{4 \mathrm{E}}-\mathrm{GST}$ and BubR1 $1_{705-1050}$ did not co-elute, indicating that they did not bind to each other (Fig. 5B). In total, our data indicate that the C-terminal helix of BubR1 is necessary but not sufficient to interact with CENP-E $2055-2358$, whereas the glutamate patch (amino acids 2313-2319) in CENP-E is essential to support the interaction.

We then tested whether CENP-E $2091-2358-$ GST-GFP could target to kinetochores in cells and whether this recruitment was only dependent on BubR1. Transiently transfected CENP$\mathrm{E}_{2091-2358}$-GST-GFP is dimeric due to the GST tag and is robustly targeted to all kinetochores; however, it did not cause chromosome misalignment (Fig. 5C), unlike CENP-E $2055-2608$. Thus the minimal kinetochore-targeting domain of CENP-E is unlikely to act as a dominant negative at kinetochores. To test whether CENP$\mathrm{E}_{2091-2358^{-}}$GST targeting to kinetochores is specifically dependent on the glutamate patch mediating interaction with BubR1, we generated CENP-E $2091-2358-4$ E - GST-GFP and hypothesized it should not be able to target to kinetochores. Indeed, CENP-E $2091-2358-4 \mathrm{E}-\mathrm{GST}-\mathrm{GFP}$ was not recruited to kinetochores (Fig. 5C,D). These data indicate that we have identified the minimal kinetochore-targeting region of CENP-E.

We next tested to what extent BubR1 contributes to CENP-E localization at kinetochores. While CENP-E is highly enriched on unattached, spindle checkpoint-active kinetochores, it is still visible on attached, metaphase kinetochores. To evaluate the contribution of BubR1 to CENP-E localization at these distinct kinetochore pools, we used defined synchronization conditions to distinguish the different kinetochore pools. In cells that had been treated with the proteasome inhibitor MG132 for $2.5 \mathrm{~h}$ to enrich for attached, spindle checkpoint-silenced kinetochores, both BubR1 and CENP-E were visible at clear, albeit modest levels in control cells (Fig. 6A; Fig. S3A). Cells depleted of BubR1 displayed a near complete loss of CENP-E from kinetochores in this situation, suggesting that CENP-E localization to microtubule-attached kinetochores is dependent on the residual pool of BubR1 retained at metaphase chromosomes (Fig. 6A-C; Fig. S3A,B). This was also the case when Bub1, essential for the recruitment of BubR1 (Johnson et al., 2004), was depleted (Fig. S3A,B). CENP-E localizes both to the outer kinetochore and to the outer corona of chromosomes, which forms preferentially on unattached kinetochores. To test whether the corona proteins are required for CENP-E kinetochore-targeting on attached kinetochores, we depleted the RZZ complex component ZW10, which is involved in corona formation (Fig. S3). ZW10 depletion did not affect CENP-E levels at attached kinetochores under our conditions. These data indicate that, although CENP-E localizes to the outer corona in a RZZ-dependent fashion, CENP-E targeting to kinetochores occurs in the absence of the RZZ complex, as shown previously (Pereira et al., 2018). In the absence of endogenous BubR1, we then expressed full-length BubR1 or BubR $1_{1-1030}$ under an inducible promoter and quantified the corresponding endogenous CENP-E at kinetochores (Fig. 6A-I). At aligned kinetochores, CENP-E levels were reduced by half in the presence of BubR $1_{1-1030}$ (Fig. 6B). CENP-E levels in the presence of BubR $1_{1-1030}$ were, however, higher than those when BubR1 was depleted (Fig. 6A-C), suggesting that BubR1 $1_{1-1030}$ enables low levels of CENP-E recruitment at kinetochores. Strikingly, when MG132-arrested cells were treated with a short $(5 \mathrm{~min})$ pulse of the microtubuledepolymerizing drug nocodazole, a method that has previously been used to test the recruitment of spindle checkpoint components to kinetochores under defined conditions (Vleugel et al., 2015), BubR1depleted or BubR $1_{1-1030}$-expressing cells were deficient in CENP-E recruitment (Fig. 6D-F). This was in clear contrast to a longer nocodazole treatment $(2.5 \mathrm{~h})$, after which the levels of CENP-E observed on BubR1-depleted and BubR1 $1-1030^{-}$-expressing kinetochores were much more similar (Fig. 6G-I). Taken together, these results suggest that BubR1 primarily facilitates initial CENP-E recruitment to spindle-assembly checkpoint (SAC)-active kinetochores and is not strictly required for CENP-E localization to this subset of kinetochores.

In the absence of BubR1, chromosomes are unable to form stable end-on attachments (Fig. 6J,K) because of the absence of BubR1recruited PP2A-B56 (Foley et al., 2011; Kruse et al., 2013; Suijkerbuijk et al., 2012). When BubR $1_{1-1030}$ was expressed in the absence of BubR1, most chromosomes were still able to form a metaphase plate, consistent with the idea that PP2A-B56 targeting was restored in this construct. However, in comparison to cells expressing GFP-BubR1 $1_{\mathrm{WT}}$, we observed a significant increase in 

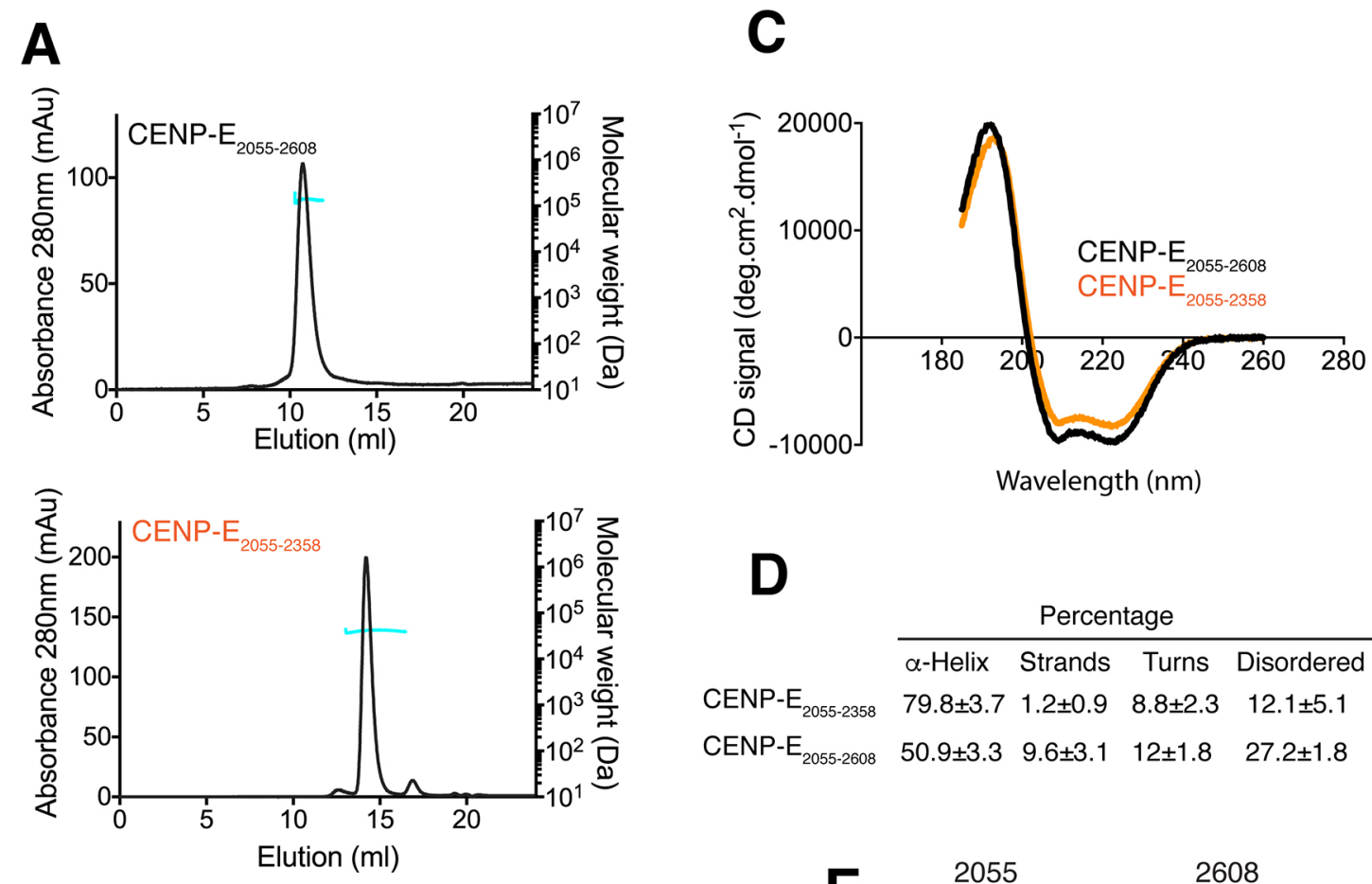

Wavelength (nm)
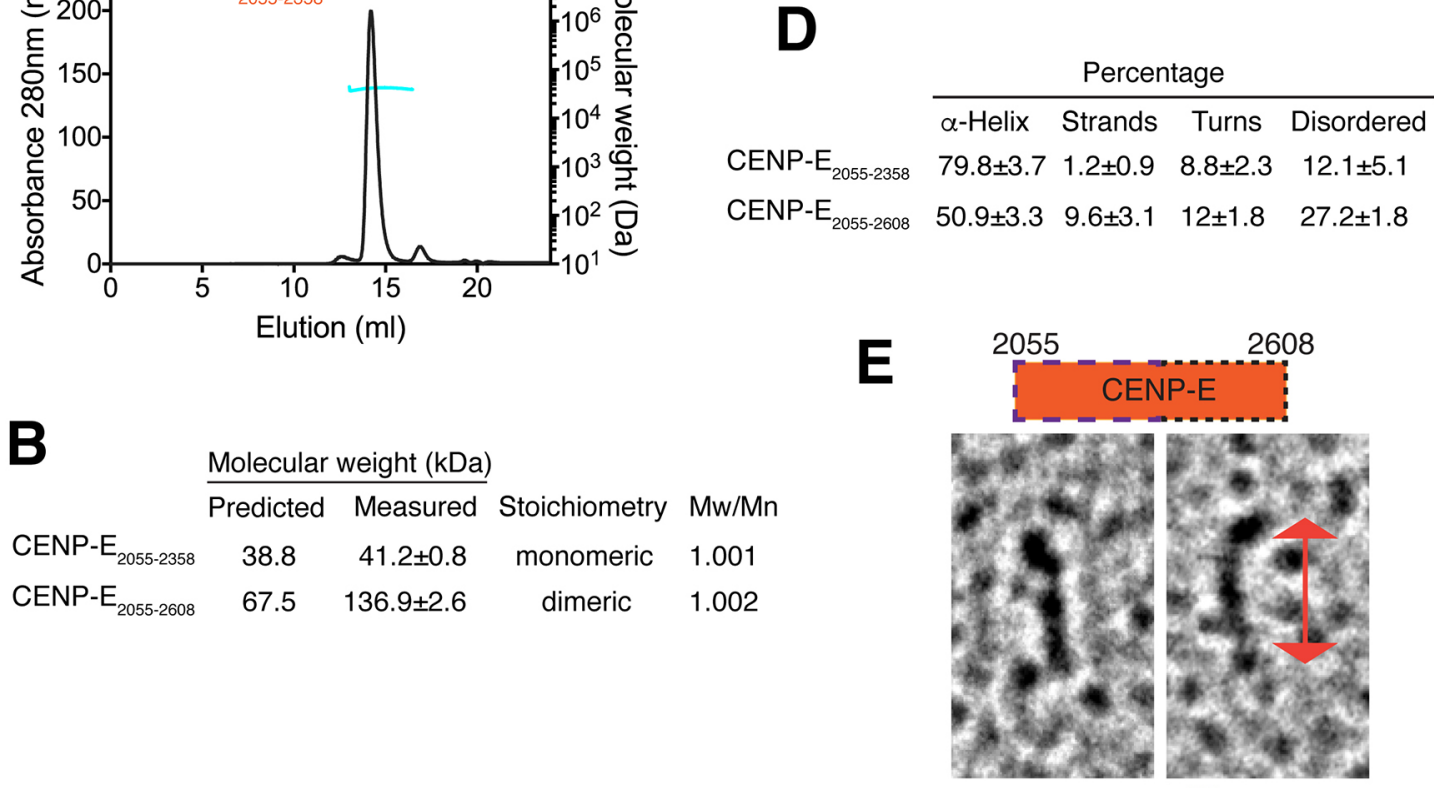

$40 \mathrm{~nm}$

Fig. 2. Biophysical analysis of CENP-E ${ }_{2055-2608}$ and $C_{\text {CNP-E }}$ 2055-2358. (A) Elution profile (black line, left $y$-axis) from a size-exclusion chromatography (SEC) run with subsequent multiangle light scattering (MALS) analysis for CENP-E ${ }_{2055-2608}$ (top) and CENP-E $2055-2358$ (bottom). Outcome of the MALS analysis for the peak is presented in blue (molecular weight, right $y$-axis). (B) Table showing the predicted and measured mass, stoichiometry of the proteins and polydispersity

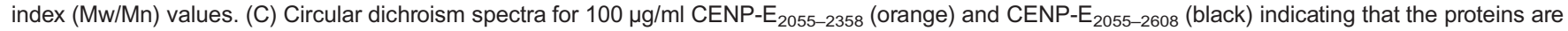
predominantly $\alpha$-helical. (D) Table summarizing the secondary structure features determined from the circular dichroism spectra in C. (E) Representative CENP$E_{2055-2608}$ particles observed after rotary shadowing. The double arrow shows the length of one particle (40 nm). Diagram shows the kinetochore-targeting domain of CENP-E. The amino acid numbers are indicated. The wide dashed line indicates the first half of the domain that binds to BubR1. The short dashed line indicates the second half of the domain, required for dimerisation.

the percentage of BubR $1_{1-1030}$ cells with misaligned chromosomes (Fig. 6K). In these cells, a small number of chromosomes were unable to congress and displayed high levels of GFP-BubR $1_{1-1030}$ at kinetochores, indicating spindle checkpoint activation (Fig. 6J,K). This phenotype is very similar to that of CENP-E depletion or knockout suggesting that a pool of CENP-E required for efficient chromosome alignment was missing (Fig. 1D) (Schaar et al., 1997). CENP-E, however, was present on the same kinetochores, recruited presumably through a pathway that does not depend on the C terminus of BubR1. Our data so far have demonstrated that the BubR1 C-terminal helix specifically recruits one pool of CENP-E to kinetochores in mitosis and during spindle checkpoint activation. This interaction seems to be required for the productive chromosome alignment and bi-orientation of chromosomes. In the absence of this specific BubR1-CENP-E interaction, the CENP-E recruitment to kinetochores through another pathway does not seem to enable full chromosome alignment. Importantly, in our experiments, the GFPBubR1 construct was expressed with levels similar to endogenous BubR1 (Fig. 6L). Overall our data indicate that BubR1 recruits CENP-E to bi-oriented chromosomes and is important for rapid recruitment of CENP-E to unattached kinetochores during SAC activation. In the absence of BubR1, another, hitherto uncharacterized pathway, also promotes CENP-E localization to kinetochores during the maintenance of SAC.

\section{DISCUSSION}

CENP-E is an essential motor, targeting to unattached kinetochores and playing a critical role in the congression, maintenance and biorientation of chromosomes (Shrestha and Draviam, 2013; Vitre et al., 2014; Wood et al., 1997). Here we show that BubR1 is a nanomolar affinity partner of CENP-E in mitosis and we reveal the molecular basis for the CENP-E-BubR1 interaction. We find that 
A
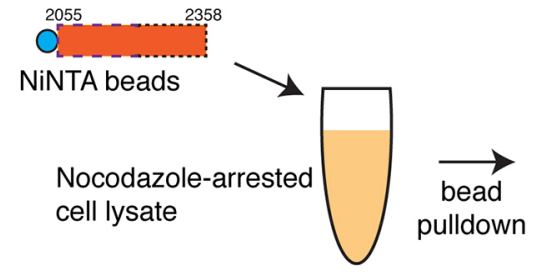

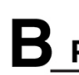

Protein MYPT1 BubR1 Centrin2 Septin 7 Spc24
MW coverage

N peptides (

$\begin{array}{lcc}8 & 109.1 & 10.3 \\ 7 & 119.6 & 10.7 \\ 5 & 19.7 & 30.8 \\ 4 & 50.6 & 15.8 \\ 1 & 17.3 & 13.9\end{array}$

D
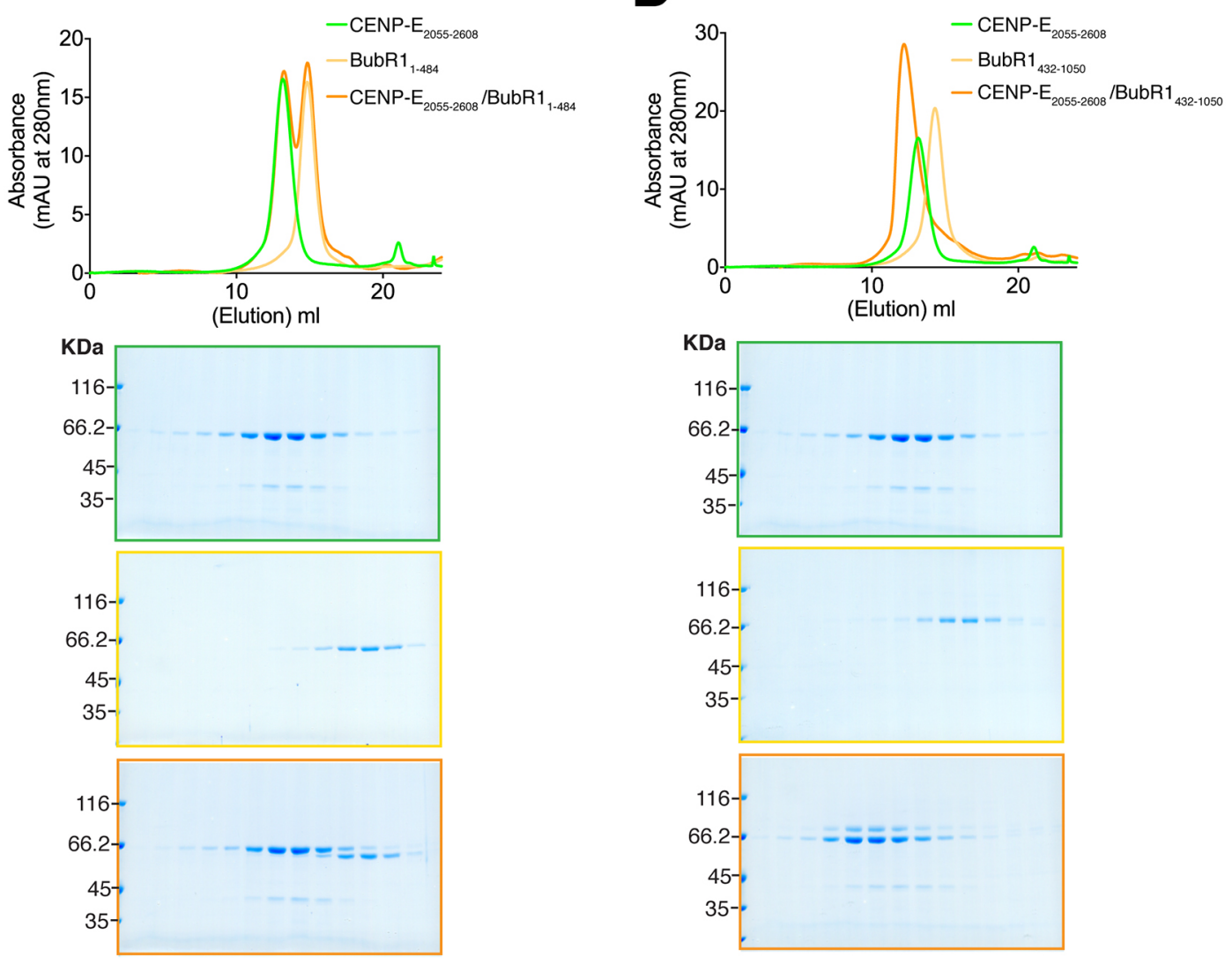

Fig. 3. The CENP-E kinetochore-targeting domain associates with the pseudokinase domain of BubR1. (A) Schematic showing the identification of CENP-

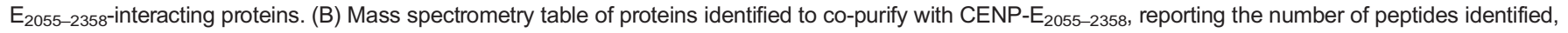
molecular weight of protein partners and their percentage peptide coverage. (C,D) Top, SEC analyses and elution profiles for the indicated constructs of CENP-E (green), BubR1 (yellow) and CENP-E-BubR1 (orange). Bottom, Coomassie-stained gels showing elution profiles for the corresponding protein complexes.

the formation of a CENP-E-BubR1 complex is not dependent on post-translational modifications. Similarly to Ciossani et al. (2018), our work indicates that the pseudokinase domain of BubR1 associates with the C-terminal kinetochore-targeting domain of CENP-E. However, this previous work used a construct that also has the centrosome-targeting domain (Fig. S1C) and a second microtubule-binding site (Ciossani et al., 2018; Gudimchuk et al., 2013). In this study, we map the domain of CENP-E necessary for kinetochore targeting. This domain is monomeric and associates with a 1:1 stoichiometry with BubR1, suggesting that full-length CENP-E can associate with two molecules of BubR1 at one time. Our data reveal BubR1 relies on its divergent and basic C-terminal helix for CENP-E binding, creating a unique and specific association to the mitotic motor. Yet, we find that this helix is not sufficient; on CENP-E a small acidic patch is critical to specify the interaction with BubR1. Mutation of these amino acids prevents the targeting of this CENP-E $2055-2358$ domain to kinetochores.
Previous work on how CENP-E localizes to kinetochores remains unclear, and the extent to which CENP-E requires BubR1 is conflicting. It is likely due to experimental differences between protocols. Indeed, our data suggest that BubR1 primarily facilitates the rapid and initial recruitment of CENP-E to kinetochores at the onset of SAC signaling. Once the SAC is 'on' for a significant period of time, we find CENP-E levels become identical at kinetochores in the presence or absence of BubR1, in good agreement with previous work (Ciossani et al., 2018). Our data indicate that BubR1 is a major interactor of CENP-E at kinetochores but there are distinct yet redundant pathways to recruit CENP-E. BubR1 increases the kinetics of CENP-E recruitment to kinetochores during spindle checkpoint activation. The other pathways contribute to a slower but robust targeting of CENP-E to kinetochores. However, they are not sufficient to restore the CENP-E function in chromosome alignment. We therefore suggest that the BubR1-dependent recruitment of CENP-E to kinetochores 

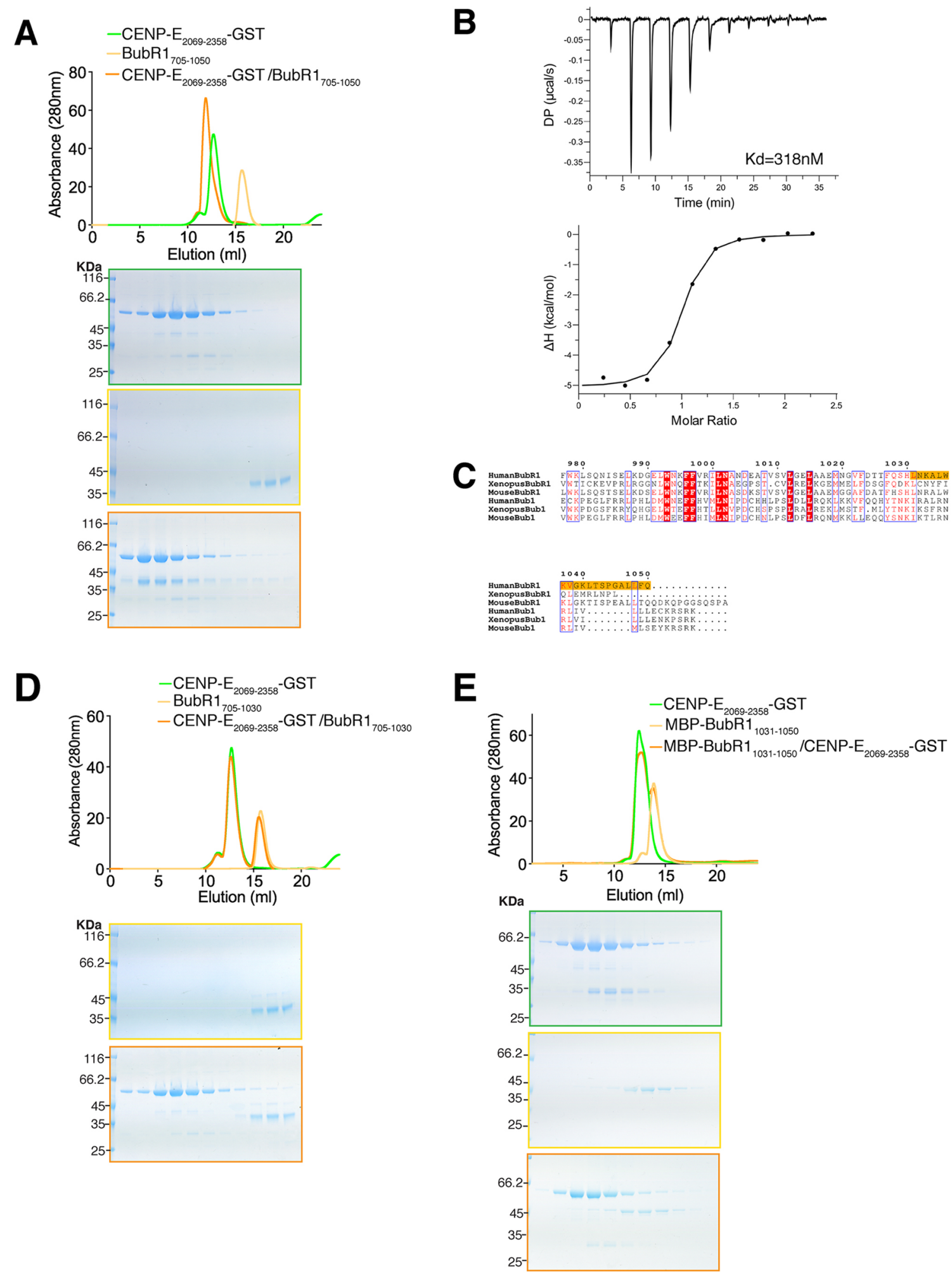

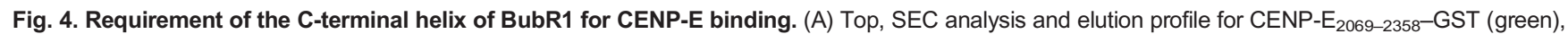
BubR1 $_{705-1050}$ (yellow) and CENP-E 2069-2358-GST-BubR1 $_{705-1050}$ (orange). Bottom, Coomassie-stained gels showing elution profiles for the corresponding protein

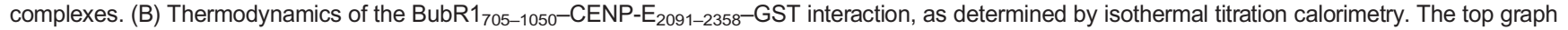
shows the differential power (DP, $\mu \mathrm{cal} / \mathrm{s}$ ) at each injection (peak). The time of each injection is shown on the $x$-axis. In the bottom graph, the peaks were integrated and are displayed in a Wiseman plot, which shows the enthalpy $(\Delta \mathrm{H})$ of each titration on the $y$-axis and the molar ratio on the $x$-axis. The $y$-axis indicates $\mathrm{kcal} / \mathrm{mol}$ of injectant. The dissociation constant $\left(K_{\mathrm{d}}\right)$ between BubR1 $1_{705-1050}$ and CENP-E $E_{2091-2358}-\mathrm{GST}$ was determined to be $318 \pm 90 \mathrm{nM}$ (mean $\pm \mathrm{s}$.d.). (C) Sequence alignment of the $\mathrm{C}$ termini of human, mouse and Xenopus Bub1 and BubR1. Boxed red and blue are the conserved and similar amino acids across all six proteins, respectively. Amino acids in red are those with conserved properties. The sequence necessary for BubR1 binding to CENP-E $E_{2055-2608}$ is highlighted in orange. (D,E) SEC analysis and elution profile for CENP-E $2069-235{ }^{-G S T}$ (green), BubR1 $1_{705-1030}$ and MBP-BubR1 $1_{1031-1050}$ (yellow in C and D, respectively), and CENP$E_{2069-2358}-G S T-B u b R 1$ constructs (orange). Bottom, Coomassie-stained gels showing elution profiles for the corresponding protein complexes. 
A

Homo_sapiens Mus_musculus Pan_troglodyte Pongo_abelii Physeter_catodon Octodon_degus Equus_caballus Pteropus_vampyrus Meriones_unguiculatus

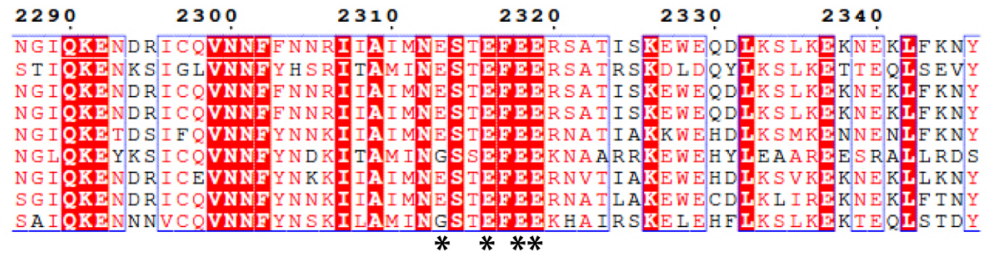

B
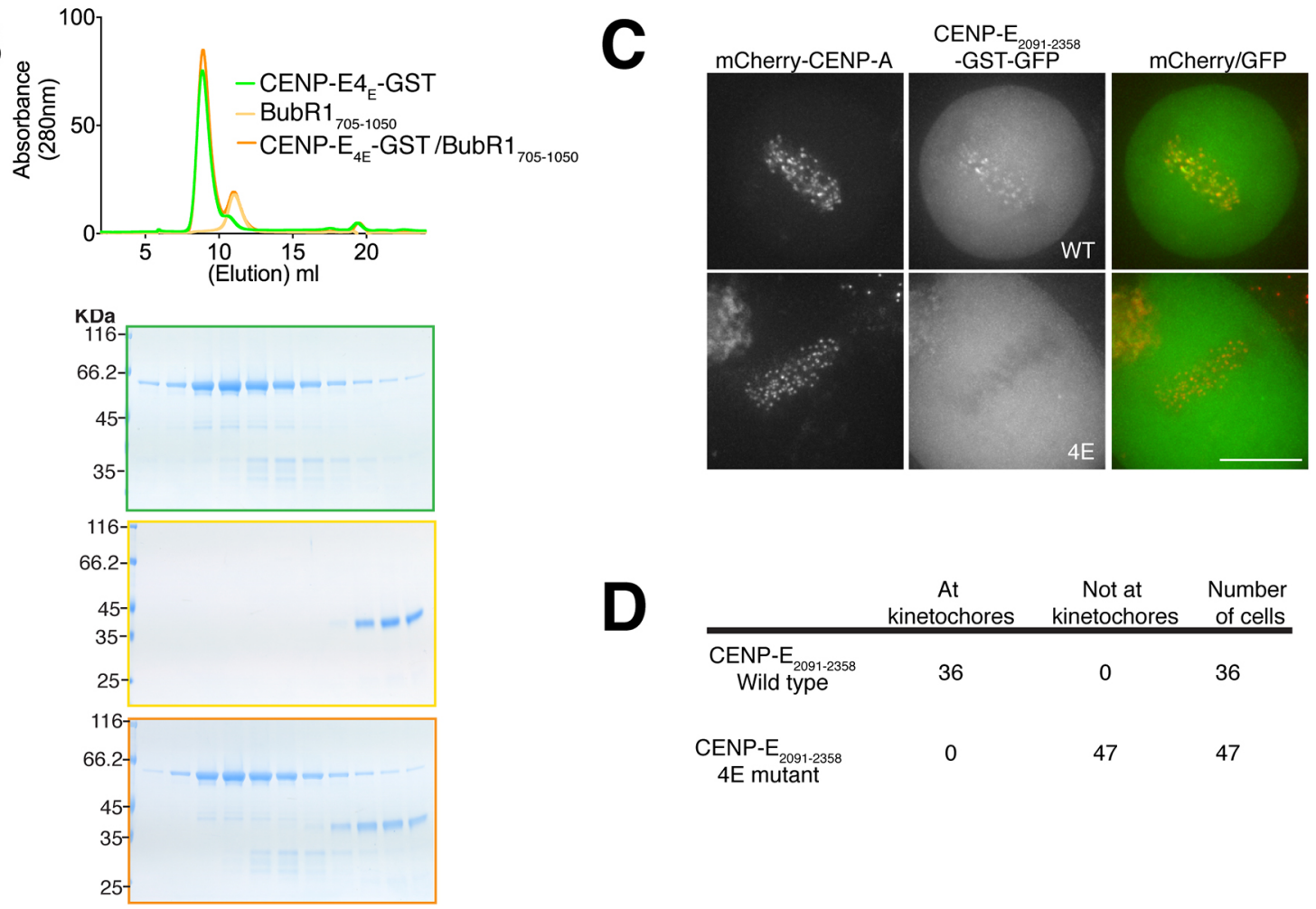

D

\begin{tabular}{cccc} 
& $\begin{array}{c}\text { At } \\
\text { kinetochores }\end{array}$ & $\begin{array}{c}\text { Not at } \\
\text { kinetochores }\end{array}$ & $\begin{array}{c}\text { Number } \\
\text { of cells }\end{array}$ \\
\hline $\begin{array}{c}\text { CENP- } E_{2001-2358} \\
\text { Wild type }\end{array}$ & 36 & 0 & 36 \\
$\begin{array}{c}\text { CENP-E } \\
\text { 4E mutant }\end{array}$ & 0 & 47 & 47
\end{tabular}

Fig. 5. CENP-E uses an acidic patch to bind BubR1. (A) Sequence alignment of human CENP-E $2287-2246$ with mouse, chimpanzee, orangutan, sperm whale, degu, horse, flying fox and gerbil CENP-E sequences. Boxed red and blue are the conserved and similar amino acids across all species, respectively. Amino

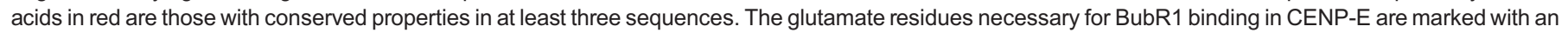

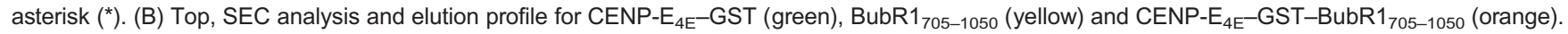

Bottom, Coomassie-stained gels showing elution profiles for the corresponding protein complexes. (C) Images of live HeLa cells expressing mCherry-CENP-A, transfected with CENP-E $2091-2358-G S T-G F P(W T)$ and CENP-E $2091-2358-4 E-G S T-G F P(4 E)$ constructs. Scale bar: $10 \mu m$. (D) Quantification of the targeting to kinetochores for CENP-E $2091-2358-G S T-G F P$ and CENP-E $2091-2358-4 E^{-G S T-G F P . ~}$

is particularly important for correct bi-orientation of kinetochores. In addition to aiding its localization, BubR1 might also regulate CENP-E activity at kinetochores, as has been recently suggested (Huang et al., 2019). In the absence of this BubR1-associated pool of CENP-E at kinetochores, the kinetochore-microtubule attachment was compromised, even when CENP-E molecules are recruited via a distinct pathway (Fig. 6J,K). Our work will now further facilitate the identification of BubR1-independent pathways that allow CENP-E to associate with kinetochores, and to define the contribution of this CENP-E pool to chromosome alignment and bi-orientation.

\section{MATERIALS AND METHODS \\ Cloning}

To assay the localization in cell culture of CENP-E subdomains, various constructs were generated from CENP-E transcript variant 1 (NM_001813. 2) and cloned into pBABE-puro containing an N-or C-terminal GFP tag and using restriction enzymes (Cheeseman and Desai, 2005). Bacteriallyexpressed constructs were cloned in pET-3aTr (Tan, 2001). pFL MultiBac
His-BubR1:Bub3 was a kind gift from Andrea Musacchio (Max Planck Institute of Molecular Physiology, Dortmund, Germany).

\section{Protein expression, purification and assays}

All constructs for bacterial expression were transformed in E. coli BL21CodonPlus (DE3)-RIL (Agilent Technologies). Cultures were induced with $0.5 \mathrm{mM}$ IPTG when $\mathrm{OD}_{600}=0.6$ for $4 \mathrm{~h}$ at $25^{\circ} \mathrm{C}$, or overnight at $18^{\circ} \mathrm{C}$ for BubR $1_{705-1050}$. Cells were resuspended in lysis buffer (50 mM HEPES $\mathrm{pH} 7.5,500 \mathrm{mM} \mathrm{NaCl}, 40 \mathrm{mM}$ imidazole, $1 \mathrm{mM}$ EDTA and $5 \mathrm{mM}$ $\beta$-Mercaptoethanol) supplemented with $1 \mathrm{mM}$ PMSF and cOmplete EDTA-free protease inhibitor cocktail (Roche), and lysed by sonication. The lysate was cleared by centrifugation ( $50 \mathrm{~min}, 58,540 \mathrm{~g}, 22,000$ r.p.m.) in a JA 25.50 rotor (Beckman Coulter), filtered and loaded onto a HisTrap HP column (GE Healthcare). Proteins were eluted in elution buffer (lysis buffer with $500 \mathrm{mM}$ imidazole). Constructs containing a $3 \mathrm{C}$ protease cleavage site were incubated overnight in dialysis buffer (25 mM HEPES pH 7.5, $300 \mathrm{mM}$ $\mathrm{NaCl}, 10 \mathrm{mM}$ imidazole, $1 \mathrm{mM}$ EDTA and $5 \mathrm{mM} \beta$-Mercaptoethanol) with $3 \mathrm{C}$ protease and then loaded onto a HisTrap HP column (GE Healthcare). The protein was then concentrated and loaded on a Superdex 200 Increase 10/300 GL (GE Healthcare) pre-equilibrated in size-exclusion chromatography buffer 
A

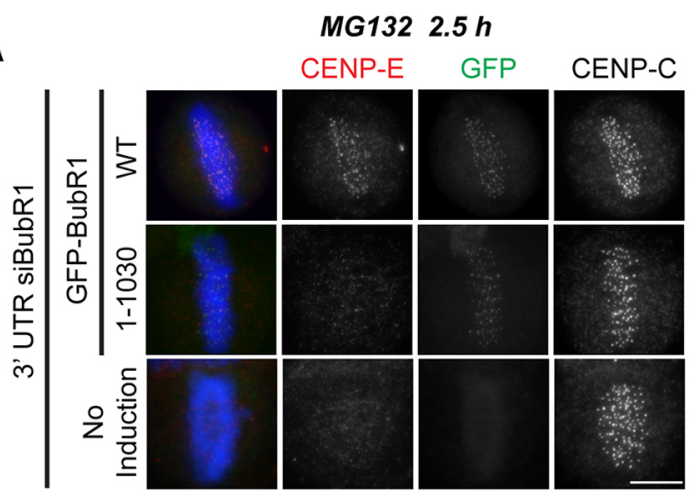

D

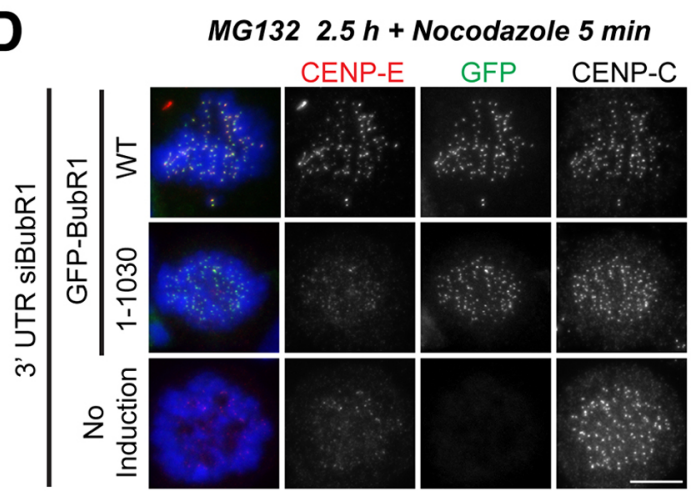

G
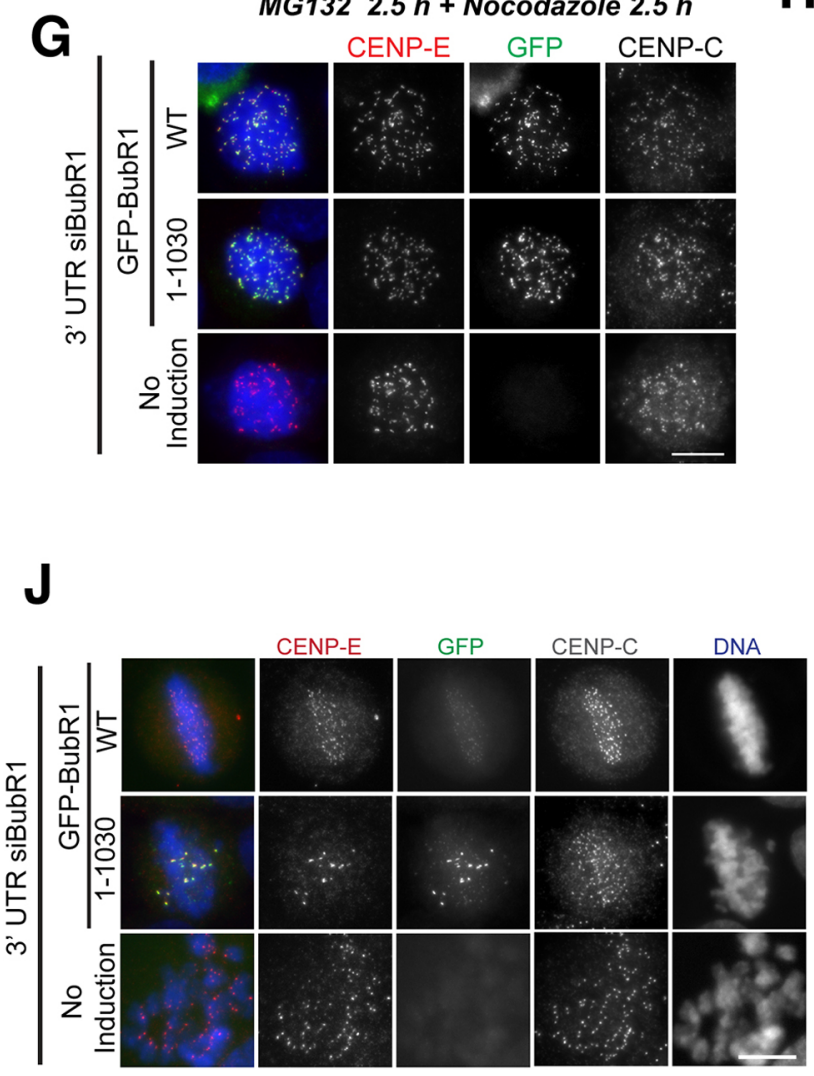

B
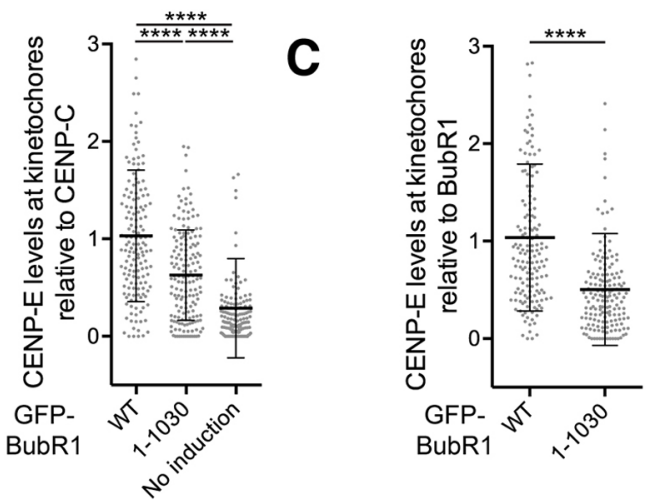

E
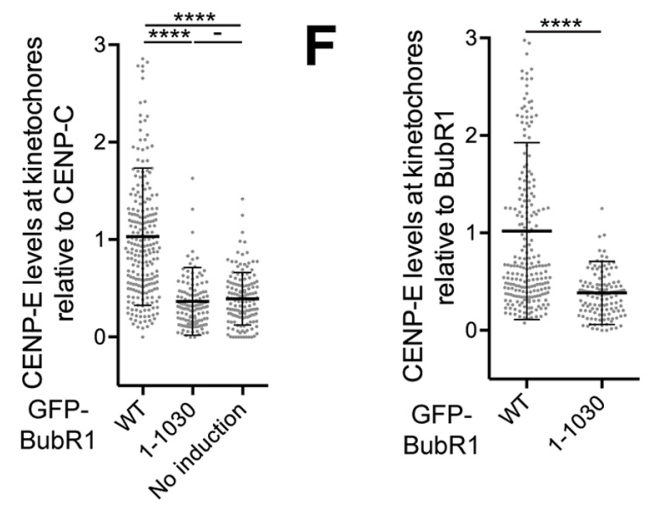

H
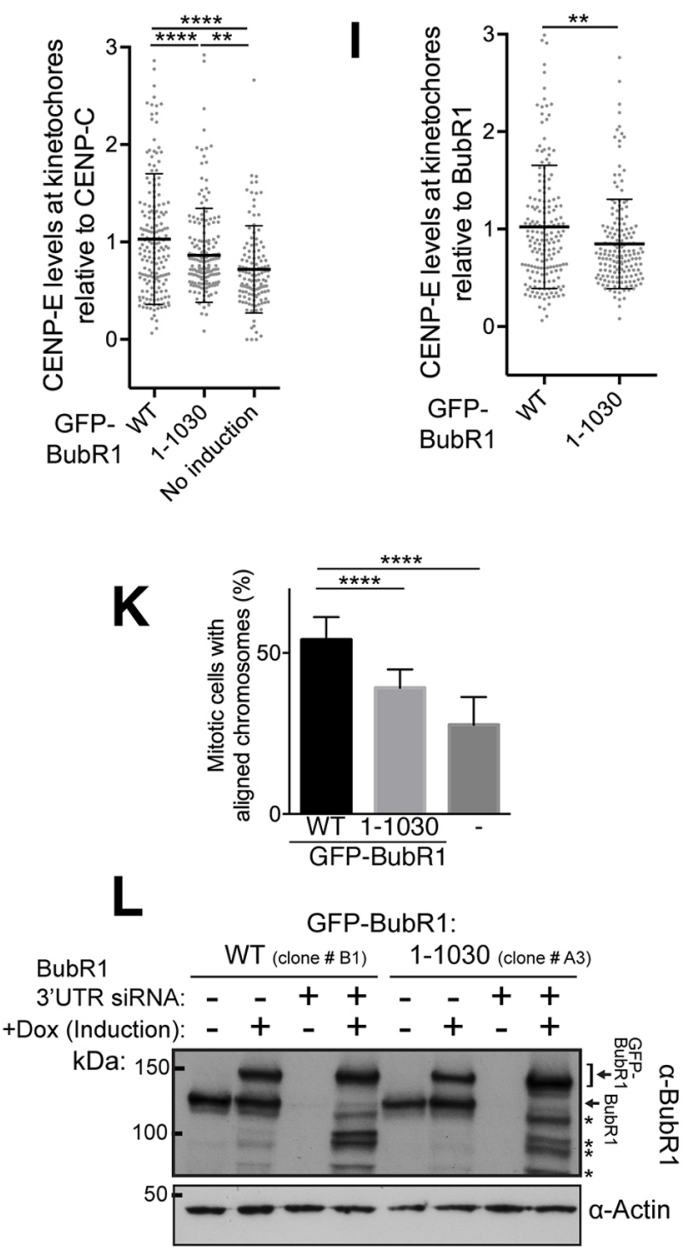

Fig. 6. See next page for legend. 
Fig. 6. BubR1 dependency of CENP-E recruitment to kinetochores and chromosome alignment. (A) Representative immunofluorescence images of HeLa cells treated with BubR1 siRNA and induced to express wild-type GFP_ BubR1 (WT) and GFP-BubR1 ${ }_{1-1030}$, stained with CENP-E, CENP-C and Hoechst after treatment with MG132 for $2.5 \mathrm{~h}$. No induction: cells with endogenous BubR1 depleted but no induction of the GFP-tagged construct. $(B, C)$ Scatter plots showing CENP-E intensity relative to CENP-C $(B)$ or GFP-BubR1 (C) in cells expressing GFP-BubR1 (WT; $n=154$ kinetochores) or GFP-BubR $1_{1-1030}$ (1-1030; $n=162$ kinetochores), and cells with endogenous BubR1 depleted but no GFP induced (no induction; $n=135$ kinetochores). (D) Same as in A. Cells were treated with MG132 for $2.5 \mathrm{~h}$ and nocodazole for 5 min. $(E, F)$ Scatter plots showing CENP-E intensity relative to CENP-C (E) or GFP-BubR1 (F), with cells expressing either GFP-BubR1 (WT; $n=228$ kinetochores) or GFP-BubR1 ${ }_{1-1030}$ (1-1030; $n=133$ kinetochores), and cells with endogenous BubR1 depleted but no GFP induced (no induction; $n=140$ kinetochores). (G) Same as in A. Cells were treated with MG132 and nocodazole for $2.5 \mathrm{~h}$. $(\mathrm{H}, \mathrm{I})$ Scatter plots showing CENP-E intensity relative to CENP-C (H) or GFP-BubR1 (I), with cells expressing either GFP-BubR1 (WT; $n=176$ kinetochores) or GFP-BubR $1_{1-1030}(1-1030 ; n=166$ kinetochores), and cells with endogenous BubR1 depleted but no GFP induced (no induction; $n=131$ kinetochores). For A-I, individual kinetochores at the metaphase plate are plotted as grey circles, with mean \pm s.d. represented by black lines. Ratios are normalized to the mean value of GFP-BubR1 WT. CENP-E:BubR1 ratios were tested using a Student's $t$-test: ${ }^{* * *} P<0.0001 ;{ }^{* *} P=0.0049 ;-, P>0.05$. Measurements were carried out across two independent experiments. (J) Representative immunofluorescence images of HeLa cells treated with BubR1 siRNA and induced to express GFP-BubR1 WT or GFP-BubR1 1-1030, stained with CENP-E, CENP-C and Hoechst after treatment with MG132 for $2 \mathrm{~h}$. (K) Graph showing percentage of cells with at least one misaligned chromosome for BubR1-depleted cells induced to express GFP-BubR1, GFP-BubR1 $1_{1-1030}$, or without induction. Data are mean \pm s.d. Measurements were carried out across two independent experiments. ${ }^{* * \star *} P<0.0001$ (one-way ANOVA). (L) Western blot for cells in this figure, probed for BubR1 and actin as a loading control. Upper arrow indicates GFP-BubR1 bands, lower arrow indicates endogenous BubR1 band, * indicates GFP-BubR1 degradation products. Scale bars: $10 \mu \mathrm{m}$.

[20 mM HEPES pH 7.5, $300 \mathrm{mM} \mathrm{NaCl}$ (or $500 \mathrm{mM}$ for CENP-E ${ }_{2055-2608}$ ), $1 \mathrm{mM}$ EDTA and $1 \mathrm{mM}$ dithiothreitol (DTT)].

Constructs for insect cell expression were transfected and expressed in SF9 cells using the Bac-to-Bac ${ }^{\mathbb{R}}$ expression system (Thermo Fisher Scientific). Expression was carried out for $72 \mathrm{~h}$ at $27^{\circ} \mathrm{C}$. Cells were resuspended in lysis buffer supplemented with $1 \mathrm{mM}$ PMSF and cOmplete EDTA-free protease inhibitor cocktail (Roche) and lysed by sonication. The lysate was cleared by centrifugation ( $60 \mathrm{~min}, 125,440 \mathrm{~g}, 40,000$ r.p.m.) in a Type 45 Ti rotor (Beckman Coulter), filtered and loaded onto a HisTrap HP column (GE Healthcare). Proteins were eluted and purified by sizeexclusion chromatography as for the constructs expressed in bacteria.

Bacteria expressing MBP-BubR1 $1_{1031-1050}$ were resuspended in MBP lysis buffer $(50 \mathrm{mM}$ HEPES $\mathrm{pH} 7.5,500 \mathrm{mM} \mathrm{NaCl}, 1 \mathrm{mM}$ EDTA and $1 \mathrm{mM}$ DTT) supplemented with $1 \mathrm{mM}$ PMSF and cOmplete EDTA-free protease inhibitor cocktail (Roche) and lysed by sonication. The lysate was cleared by centrifugation ( $50 \mathrm{~min}, 58,540 \mathrm{~g}, 22,000$ r.p.m.) in a JA 25.50 rotor (Beckman Coulter), filtered and loaded onto an MBPTrap HP column (GE Healthcare). Proteins were eluted in elution buffer (lysis buffer with $10 \mathrm{mM}$ maltose). The fractions containing the protein were concentrated and gel filtered on a Superdex 75 increase 10/300 GL (GE Healthcare) preequilibrated in size-exclusion chromatography buffer.

For ITC, CENP-E $2091-2358$-GST was purified in complex with $6 \times$ HisBubR $1_{705-1050}$. Both lysates were mixed, cleared by centrifugation, filtered and loaded onto a HisTrap HP column. After overnight incubation with $3 \mathrm{C}$ protease, the complex was further purified on a Superdex Increase 20010 / 300 GL column (GE Healthcare) in separation buffer (50 mM HEPES pH $7.5,800 \mathrm{mM} \mathrm{NaCl}, 1 \mathrm{mM}$ EDTA and $1 \mathrm{mM}$ DTT). The fractions containing CENP-E ${ }_{2091-2358}-$ GST and BubR $1_{705-1050}$ were pooled independently then dialysed against the ITC buffer. All binding assays were carried out on a Superdex Increase $20010 / 300$ GL column in binding buffer $(20 \mathrm{mM}$ HEPES pH 7.5, $150 \mathrm{mM} \mathrm{NaCl}, 1 \mathrm{mM}$ EDTA and $1 \mathrm{mM}$ DTT). Proteins were mixed in equimolar ratio at $\sim 7 \mu \mathrm{M}$.

\section{Size-exclusion chromatography coupled to multiangle light scattering}

Size-exclusion chromatography (ÄKTA PURETM, GE Healthcare) coupled to UV, static light scattering and refractive index detection (Viscotec SECMALS 20 and Viscotek RI Detector VE3580, Malvern Instruments) were used to determine the absolute molecular mass of the indicated proteins in solution. $100 \mu \mathrm{l}$ of CENP-E $2055-2608$ and CENP-E $2055-2358$ at $1 \mathrm{mg} \mathrm{ml}^{-1}$ were run on a calibrated Superdex-200 10/300 GL Increase (GE Healthcare) size exclusion column pre-equilibrated in gel filtration buffer (described above) at $22^{\circ} \mathrm{C}$ with a flow rate of $1.0 \mathrm{ml} \mathrm{min}{ }^{-1}$. Light scattering, refractive index (RI) and $\mathrm{A}_{280 \mathrm{~nm}}$ were analysed using a homo-polymer model (OmniSEC software, v5.02; Malvern Instruments) using the following parameters: $\partial \mathrm{A}_{280 \mathrm{~nm}} / \partial \mathrm{c}=0.429 \mathrm{AU} \mathrm{ml} \mathrm{mg} \mathrm{m}^{-1}$ and $0.530 \mathrm{AU} \mathrm{ml} \mathrm{mg}{ }^{-1}$ for CENP-E ${ }_{2055-2608}$ and CENP-E $2055-2358$, respectively; $\partial \mathrm{n} / \partial \mathrm{c}=0.185 \mathrm{ml} \mathrm{g}^{-1}$; and a buffer RI value of 1.336 .

\section{Pulldown with CENP-E ${ }_{2055-2358}$}

Cleared mitotic lysate was obtained from 30 confluent $15-\mathrm{cm}$ dishes with HeLa cells arrested in nocodazole $(100 \mathrm{ng} / \mathrm{ml})$ for $14 \mathrm{~h}$. Ni-NTA agarose beads (NEB) alone (control) or bound to $600 \mu \mathrm{g}$ of recombinant CENP-

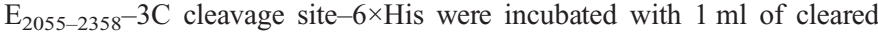
mitotic lysate for $1 \mathrm{~h}$ at $4^{\circ} \mathrm{C}$. Beads were spun down and washed in lysis buffer (50 mM HEPES pH 7.5, $1 \mathrm{mM}$ EGTA, $1 \mathrm{mM} \mathrm{MgCl} 2,100 \mathrm{mM} \mathrm{KCl}$, $10 \%$ glycerol and $0.05 \%$ NP- 40 ) three times. Beads were then incubated with $3 \mathrm{C}$ protease at room temperature for $2 \mathrm{~h}$. The supernatant was collected and proteins were precipitated with trichloroacetic acid (TCA) overnight. Precipitated proteins were then washed twice with acetone and proteolytic digestion was carried out with Trypsin (Promega) and Lys-C (Roche) according to the manufacturers' guidelines.

\section{Mass spectrometry}

Following digestion, samples were acidified with $10 \%$ trifluoroacetic acid (TFA) until $\mathrm{pH}<2.5$ and spun onto StageTips, as described previously (Rappsilber et al., 2003). Peptides were eluted in $40 \mu \mathrm{l}$ of $80 \%$ acetonitrile in $0.1 \%$ TFA and concentrated down to $1 \mu \mathrm{l}$ by vacuum centrifugation (Concentrator 5301, Eppendorf, UK). Samples were then prepared for liquid chromatography-tandem mass spectrometry (LC-MS/MS) analysis by diluting them to $5 \mu \mathrm{l}$ with $0.1 \%$ TFA. LC-MS/MS analyses were performed on a Q Exactive mass spectrometer (Thermo Fisher Scientific, UK) coupled on-line to an Ultimate 3000 RSLCnano System (Dionex, Thermo Fisher Scientific, UK). Peptides were separated on a $50 \mathrm{~cm}$ EASYSpray column (Thermo Scientific, UK) assembled on an EASY-Spray source (Thermo Scientific, UK) and operated at $50^{\circ} \mathrm{C}$. Mobile phase A consisted of $0.1 \%$ formic acid in water, while mobile phase B consisted of $80 \%$ acetonitrile and $0.1 \%$ formic acid. Peptides were loaded onto the column at a flow rate of $0.3 \mu 1 \mathrm{~min}^{-1}$ and eluted at a flow rate of $0.2 \mu 1 \mathrm{~min}^{-1}$ according to the following gradient: $2 \%$ to $40 \%$ mobile phase B in $120 \mathrm{~min}$ and then to $95 \%$ in $11 \mathrm{~min}$. Fourier transform mass spectra (FTMS) were recorded at 70,000 resolution (scan range 350-1400 $\mathrm{m} / \mathrm{z}$ ) and the ten most intense peaks with charge between 2 and 6 of the MS scan were selected for fragmentation with an isolation window of 2.0 Thomson for MS2 (filling $1.0 \times 10^{6}$ ions for MS scan, $5.0 \times 10^{4}$ ions for MS2, maximum fill time $60 \mathrm{~ms}$, dynamic exclusion for $50 \mathrm{~s}$ )

The MaxQuant software platform (Cox and Mann, 2008) version 1.5.2.8 was used to process raw files and the search was conducted against Homo sapiens complete/reference proteome set of Uniprot database (released in February, 2016), using the Andromeda search engine (Cox et al., 2011). The first search peptide tolerance was set to 20 p.p.m. while the main search peptide tolerance was set to 4.5 p.p.m. Isotope mass tolerance was set to 2 p.p.m. and maximum charge to 7. A maximum of two missed cleavages were allowed. Carbamidomethylation of cysteine was set as fixed modification. Oxidation of methionine and acetylation of the $\mathrm{N}$ terminus were set as variable modifications.

\section{Circular dichroism}

Circular dichroism (CD) spectra in the far-ultraviolet region $(185-260 \mathrm{~nm})$ for CENP-E $2055-2358$ and CENP-E $2055-2608(0.1 \mathrm{mg} / \mathrm{ml})$ in CD buffer 
(10 $\mathrm{mM}$ potassium phosphate $\mathrm{pH} 7.5,200 \mathrm{mM} \mathrm{NaF}$ and $0.5 \mathrm{mM} \mathrm{DTT}$ ) were recorded using a $\mathrm{CD}$ spectrometer (Jasco-J-810) at $10^{\circ} \mathrm{C}(1 \mathrm{~mm}$ path length quartz cell). Data were analysed using DichroWeb (http://dichroweb.cryst. bbk.ac.uk; Whitmore and Wallace, 2008).

\section{Isothermal titration calorimetry}

Isothermal titration calorimetry (ITC) experiments were carried out to determine the affinity and stoichiometry of the BubR1-CENP-E complex. BubR $1_{705-1050}$ and CENP-E ${ }_{2091-2358}-$ GST were extensively dialysed into ITC buffer (20 mM HEPES pH 7.5, $150 \mathrm{mM} \mathrm{NaCl}, 1 \mathrm{mM}$ EDTA, $0.005 \%$ Tween-20 and $0.5 \mathrm{mM}$ TCEP) prior to the experiment to minimize heats of dilution upon titration. Protein concentrations were determined by absorption at $280 \mathrm{~nm}$; extinction coefficients $\varepsilon$ for BubR $1_{705-1050}$ and CENP-E 2091-2358-GST were $63,370 \mathrm{M}^{-1} \mathrm{~cm}^{-1}$ and $62,800 \mathrm{M}^{-1} \mathrm{~cm}^{-1}$, respectively. $276 \mu \mathrm{M}$ BubR $1_{705-1050}$ was titrated into $205.4 \mu \mathrm{l}$ of $25 \mu \mathrm{M}$ CENP-E ${ }_{2091-2358}-\mathrm{GST}$ at $37^{\circ} \mathrm{C}$ in 11 aliquots: 1 of $0.5 \mu \mathrm{l}$ followed by 10 of $3.8 \mu \mathrm{l}$ each. The reference power was set to $3 \mu \mathrm{cal} / \mathrm{s}$ and syringe rotation 750 r.p.m. The enthalpy of binding was analysed with correction for heat of dilution using the software package provided by the instrument manufacturer (Auto-iTC200 microcalorimeter; Malvern Instruments). Data were fitted to a simple binding model with one set of sites. The experimentally derived protein concentrations were fixed and the number of binding sites on CENP-E $(N)$, the affinity of the interaction $\left(K_{\mathrm{d}}\right)$ and the enthalpy of the interaction $(\Delta H)$ floated and solved with the LevenbergMarquardt algorithm after establishing a global minima solution with the Simplex algorithm.

\section{Low-angle rotary shadowing and electron microscopy}

CENP-E $2055-2608$ at a concentration of $100 \mu \mathrm{g} / \mathrm{ml}$ in gel filtration buffer with $30 \%$ glycerol were sprayed onto a mica sheet (TAAB). CENP-E ${ }_{2055-2608}$ was shadowed with $2.5 \mathrm{~nm}$ of platinum at $5^{\circ}$ angle and $9 \mathrm{~nm}$ of carbon using a Leica EM ACE600. Replicas were detached in water and placed on non-coated grids (Type 400 mesh, TAAB). Images were obtained using a JEM-1400Plus transmission electron microscope (JEOL) operated at $90 \mathrm{kV}$. Electron micrographs were acquired using a GATAN OneView camera.

\section{Cell culture and experiments}

HeLa CCL2 cells, from ATCC, were used and maintained in DMEM (Lonza) supplemented with $5 \% \mathrm{CO}_{2}$ at $37^{\circ} \mathrm{C}$ in a humidified atmosphere. The inducible HeLa Cas9 sgRNA CENP-E cell line was obtained from Iain Cheeseman (Whitehead Institute, Cambridge, MA, USA; McKinley and Cheeseman, 2017) and maintained in a tetracycline-free medium. Cells were checked monthly for mycoplasma contamination (MycoAlert detection kit, Lonza). Transient transfections were conducted using Effectene reagent (Qiagen) according to the manufacturer's guidelines. GFP-BubR1 wild type (WT) and 1-1030 HeLa cell lines (\#B1 and \#A3, respectively) were generated with single integrated copies of the desired transgenes using the T-Rex doxycycline-inducible Flp-In system, and were chosen for equal expression levels, as seen by immunofluorescence and western blotting.

GFP-BubR1 was induced $6 \mathrm{~h}$ before a 48 -h siRNA depletion of endogenous BubR1 using oligonucleotides against the 3' UTR (5'-GCAATCAAGTCTCACAGAT-3') (Espert et al., 2014). A second induction was performed $24 \mathrm{~h}$ into the siRNA depletion. $26.5 \mathrm{~h}$ prior to fixing, cells were subjected to thymidine arrest for $16 \mathrm{~h}$ followed by a $10.5 \mathrm{~h}$ release. For the final $2.5 \mathrm{~h}, \mathrm{MG} 132$ was added at $20 \mu \mathrm{M}$ to increase the metaphase population.

\section{CRISPR Cas9 knockout}

To induce Cas 9 expression, cells were treated with $1 \mu \mathrm{g} / \mathrm{ml}$ doxycycline (Sigma Aldrich) for 48-72 h, changing the medium with fresh doxycycline every $24 \mathrm{~h}$ to induce the knockout.

\section{Microscopy}

For live-cell imaging, HeLa cells were imaged in Leibovitz L15 medium or DMEM (Life Technologies) supplemented with 10\% FBS and penicillin/ streptomycin (Gibco) at $37^{\circ} \mathrm{C}$ using a Deltavision core microscope (Applied Precision) equipped with a CoolSnap HQ2 CCD camera. 4-10 $z$-sections were acquired at $0.5 \mu \mathrm{m}$ steps using a $60 \times$ objective lens. For immunofluorescence, cells were washed with PBS and fixed by one of two methods, either fixed in cold methanol for $10 \mathrm{~min}$ at $-20^{\circ} \mathrm{C}$ and then permeabilized with cold acetone for $1 \mathrm{~min}$ at $-20^{\circ} \mathrm{C}$, or pre-extracted with $0.4 \%$ Triton- $X$ in PHEM buffer ( $60 \mathrm{mM}$ Pipes, $25 \mathrm{mM}$ HEPES, $10 \mathrm{mM}$ EGTA, $2 \mathrm{mM} \mathrm{MgSO}_{4}, \mathrm{pH} 7.0$ ) for $1 \mathrm{~min}$ and then fixed with $3.8 \%$ formaldehyde in PHEM buffer for 20 min. For experiments with HeLa FlipIn inducible cells, fixation was performed with PTEMF (20 mM Pipes$\mathrm{KOH}, \mathrm{pH} 6.8,0.2 \%$ Triton X-100, $1 \mathrm{mM} \mathrm{MgCl}_{2}, 10 \mathrm{mM}$ EGTA and $4 \%$ formaldehyde) for $12 \mathrm{~min}$. Immunofluorescence in human cells was conducted using mouse anti- $\alpha$-tubulin (Sigma; 1:1000), mouse antiCENP-E (Abcam, Ab5093; 1:1000 or 1:200), rabbit anti-Centrin (kind gift from Iain Cheeseman; 1:1000), guinea pig anti-CENP-C (pAb; MBL PD030; 1:2000) antibodies and human ACA (anti-centromere antibodies; Cambridge Biosciences; 1:100). Hoechst 33342 (Thermo Fisher Scientific; H3570) was used to stain DNA. Nocodazole was used at final concentrations of $0.3 \mu \mathrm{M}$ for $2.5 \mathrm{~h}$. For the brief nocodazole treatment, a concentration of 3.3 $\mu \mathrm{M}$ was used. A widefield Eclipse Ti2 (Nikon) microscope equipped with a Prime 95B Scientific CMOS camera (Photometrics) was used for imaging. $Z$-sections were acquired at $0.2-\mu \mathrm{m}$ step size. Images were stored and vizualized using an OMERO.insight client (OME) (Allan et al., 2012). Mean kinetochore fluorescence intensity within a circular region of interest (ROI) with a 10 pixel diameter was measured, with a background intensity recorded in an adjacent cytoplasmic area. Relative CENP-E and BubR1 values for each kinetochore were calculated by subtracting the background values and dividing them by the background corrected ACA or CENP-C signal for that kinetochore. Data was analysed using ImageJ (Schneider et al., 2012).

\section{Statistics and reproducibility}

Statistical analyses were performed using GraphPad Prism 6.0. No statistical method was used to predetermine sample size. All experiments were performed and quantified from at least three independent experiments, unless specified and the representative data are shown.

\section{Acknowledgements}

The Wellcome Centre for Cell Biology is supported by core funding from the Wellcome Trust (203149), a multi-user equipment grant (101527) for the Edinburgh Protein Production Facility and a multi-user equipment grant (108504) for the Orbitrap Fusion Lumos.

\section{Competing interests}

The authors declare no competing or financial interests.

\section{Author contributions}

Conceptualization: T.L., J.P.I.W.; Validation: T.L., D.H., J.P.I.W.; Formal analysis: T.L., D.H., A.G.-K., E.A.B., C.S., U.G., J.P.I.W.; Investigation: T.L., A.G.-K., J.P.I.W.; Resources: J.P.I.W.; Writing - original draft: J.P.I.W.; Writing - review \& editing: T.L., A.G.-K., U.G., J.P.I.W.; Supervision: U.G., J.P.I.W.; Project administration: J.P.I.W.; Funding acquisition: J.R., U.G., J.P.I.W.

\section{Funding}

J.R. is supported by a Wellcome Trust Senior Research Fellowship (103139). J.P.I.W. is supported by a Wellcome Trust Senior Reseach Fellowship (207430). D.H. was supported by a Medical Research Council Senior Non-Clinical Fellowship (MR/K006703/1) awarded to U.G. Open access funding provided by University of Edinburgh. Deposited in PMC for immediate release.

\section{Supplementary information}

Supplementary information available online at

https://jcs.biologists.org/lookup/doi/10.1242/jcs.246025.supplemental

\section{Peer review history}

The peer review history is available online at

https://jcs.biologists.org/lookup/doi/10.1242/jcs.246025.reviewer-comments.pdf

\section{References}

Akera, T., Goto, Y., Sato, M., Yamamoto, M. and Watanabe, Y. (2015). Mad1 promotes chromosome congression by anchoring a kinesin motor to the kinetochore. Nat. Cell Biol. 17, 1124-1133. doi:10.1038/ncb3219

Allan, C., Burel, J. M., Moore, J., Blackburn, C., Linkert, M., Loynton, S., Macdonald, D., Moore, W. J., Neves, C., Patterson, A. et al. (2012). OMERO: flexible, model-driven data management for experimental biology. Nat. Methods 9 , 245-253. doi:10.1038/nmeth.1896 
Ashar, H. R., James, L., Gray, K., Carr, D., Black, S., Armstrong, L., Bishop W. R. and Kirschmeier, P. (2000). Farnesyl transferase inhibitors block the farnesylation of CENP-E and CENP-F and alter the association of CENP-E with the microtubules. J. Biol. Chem. 275, 30451-30457. doi:10.1074/jbc.M003469200

Brown, K. D., Wood, K. W. and Cleveland, D. W. (1996). The kinesin-like protein CENP-E is kinetochore-associated throughout poleward chromosome segregation during anaphase-A. J. Cell Sci. 109, 961-969.

Chan, G. K. T., Schaar, B. T. and Yen, T. J. (1998). Characterization of the kinetochore binding domain of CENP-E reveals interactions with the kinetochore proteins CENP-F and hBUBR1. J. Cell Biol. 143, 49-63. doi:10.1083/jcb.143.1.49

Chan, G. K. T., Jablonski, S. A., Sudakin, V., Hittle, J. C. and Yen, T. J. (1999). Human BUBR1 is a mitotic checkpoint kinase that monitors CENP-E functions at kinetochores and binds the cyclosome/APC. J. Cell Biol. 146, 941-954. doi:10. 1083/jcb.146.5.941

Cheeseman, I. M. and Desai, A. (2005). A combined approach for the localization and tandem affinity purification of protein complexes from metazoans. Sci. STKE 2005, pl1. doi:10.1126/stke.2662005pl1

Ciossani, G., Overlack, K., Petrovic, A., Huis In 't Veld, P. J., Koerner, C., Wohlgemuth, S., Maffini, S. and Musacchio, A. (2018). The kinetochore proteins CENP-E and CENP-F directly and specifically interact with distinct BUB mitotic checkpoint Ser/Thr kinases. J. Biol. Chem. 293, 10084-10101. doi:10. 1074/jbc.RA118.003154

Cooke, C. A., Schaar, B., Yen, T. J. and Earnshaw, W. C. (1997). Localization of CENP-E in the fibrous corona and outer plate of mammalian kinetochores from prometaphase through anaphase. Chromosoma 106, 446-455. doi:10.1007/ s004120050266

Cox, J. and Mann, M. (2008). MaxQuant enables high peptide identification rates, individualized p.p.b.-range mass accuracies and proteome-wide protein quantification. Nat. Biotechnol. 26, 1367-1372. doi:10.1038/nbt.1511

Cox, J., Neuhauser, N., Michalski, A., Scheltema, R. A., Olsen, J. V. and Mann, M. (2011). Andromeda: a peptide search engine integrated into the MaxQuant environment. J. Proteome Res. 10,1794-1805. doi:10.1021/pr101065

Craske, B. and Welburn, J. P. I. (2020). Leaving no-one behind: how CENP-E facilitates chromosome alignment. Essays Biochem. [Epub] EBC20190073. doi:10.1042/EBC20190073

Espert, A., Uluocak, P., Bastos, R. N., Mangat, D., Graab, P. and Gruneberg, U. (2014). PP2A-B56 opposes Mps1 phosphorylation of Knl1 and thereby promotes spindle assembly checkpoint silencing. J. Cell Biol. 206, 833-842. doi:10.1083/ jcb.201406109

Foley, E. A., Maldonado, M. and Kapoor, T. M. (2011). Formation of stable attachments between kinetochores and microtubules depends on the B56-PP2A phosphatase. Nat. Cell Biol. 13, 1265-1271. doi:10.1038/ncb2327

Gudimchuk, N., Vitre, B., Kim, Y., Kiyatkin, A., Cleveland, D. W., Ataullakhanov, F. I. and Grishchuk, E. L. (2013). Kinetochore kinesin CENP-E is a processive bidirectional tracker of dynamic microtubule tips. Nat. Cell Biol. 15, 1079-1088. doi:10.1038/ncb2831

Huang, Y., Lin, L., Liu, X., Ye, S., Yao, P. Y., Wang, W., Yang, F., Gao, X., Li, J., Zhang, Y. et al. (2019). BubR1 phosphorylates CENP-E as a switch enabling the transition from lateral association to end-on capture of spindle microtubules. Cell Res. 29, 562-578. doi:10.1038/s41422-019-0178-z

Johnson, V. L., Scott, M. I., Holt, S. V., Hussein, D. and Taylor, S. S. (2004). Bub1 is required for kinetochore localization of BubR1, Cenp-E, Cenp-F and Mad2, and chromosome congression. J. Cell Sci. 117, 1577-1589. doi:10.1242/jcs.01006

Kapoor, T. M., Lampson, M. A., Hergert, P., Cameron, L., Cimini, D., Salmon, E. D., McEwen, B. F. and Khodjakov, A. (2006). Chromosomes can congress to the metaphase plate before biorientation. Science 311, 388-391. doi:10.1126/ science.1122142

Kops, G. J. P. L., Foltz, D. R. and Cleveland, D. W. (2004). Lethality to human cancer cells through massive chromosome loss by inhibition of the mitotic checkpoint. Proc. Natl. Acad. Sci. USA 101, 8699-8704. doi:10.1073/pnas. 0401142101

Kruse, T., Zhang, G., Larsen, M. S. Y., Lischetti, T., Streicher, W., Kragh Nielsen, T., Bjorn, S. P. and Nilsson, J. (2013). Direct binding between BubR1 and B56 PP2A phosphatase complexes regulate mitotic progression. J. Cell Sci. 126, 1086-1092. doi:10.1242/jcs.122481

Maffini, S., Maia, A. R., Manning, A. L., Maliga, Z., Pereira, A. L., Junqueira, M., Shevchenko, A., Hyman, A., Yates, J. R., III, Galjart, N. et al. (2009). Motorindependent targeting of CLASPs to kinetochores by CENP-E promotes microtubule turnover and poleward flux. Curr. Biol. 19, 1566-1572. doi:10.1016/ j.cub.2009.07.059

Maiato, H., Gomes, A. M., Sousa, F. and Barisic, M. (2017). Mechanisms of chromosome congression during mitosis. Biology (Basel) 6, 13. doi:10.3390/ biology6010013
Maliga, Z., Junqueira, M., Toyoda, Y., Ettinger, A., Mora-Bermúdez, F., Klemm, R. W., Vasilj, A., Guhr, E., Ibarlucea-Benitez, I., Poser, I. et al. (2013). A genomic toolkit to investigate kinesin and myosin motor function in cells. Nat. Cell Biol. 15, 325-334. doi:10.1038/ncb2689

Mao, Y., Abrieu, A. and Cleveland, D. W. (2003). Activating and silencing the mitotic checkpoint through CENP-E-dependent activation/inactivation of BubR1. Cell 114, 87-98. doi:10.1016/S0092-8674(03)00475-6

McKinley, K. L. and Cheeseman, I. M. (2017). Large-scale analysis of CRISPR/ Cas9 cell-cycle knockouts reveals the diversity of p53-dependent responses to cell-cycle defects. Dev. Cell 40, 405-420.e402. doi:10.1016/j.devcel.2017.01.012

Pereira, C., Reis, R. M., Gama, J. B., Celestino, R., Cheerambathur, D. K. Carvalho, A. X. and Gassmann, R. (2018). Self-assembly of the RZZ complex into filaments drives kinetochore expansion in the absence of microtubule attachment. Curr. Biol. 28, 3408-3421.e3408. doi:10.1016/j.cub.2018.08.056

Raaijmakers, J. A., van Heesbeen, R., Blomen, V. A., Janssen, L. M. E., van Diemen, F., Brummelkamp, T. R. and Medema, R. H. (2018). BUB1 is essential for the viability of human cells in which the spindle assembly checkpoint is compromised. Cell Rep. 22, 1424-1438. doi:10.1016/j.celrep.2018.01.034

Rappsilber, J., Ishihama, Y. and Mann, M. (2003). Stop and go extraction tips for matrix-assisted laser desorption/ionization, nanoelectrospray, and LC/MS sample pretreatment in proteomics. Anal. Chem. 75, 663-670. doi:10.1021/ac026117i

Sacristan, C., Ahmad, M. U. D., Keller, J., Fermie, J., Groenewold, V., Tromer, E., Fish, A., Melero, R., Carazo, J. M., Klumperman, J. et al. (2018). Dynamic kinetochore size regulation promotes microtubule capture and chromosome biorientation in mitosis. Nat. Cell Biol. 20, 800-810. doi:10.1038/s41556-0180130-3

Schaar, B. T., Chan, G. K. T., Maddox, P., Salmon, E. D. and Yen, T. J. (1997) CENP-E function at kinetochores is essential for chromosome alignment. J. Cell Biol. 139, 1373-1382. doi:10.1083/jcb.139.6.1373

Schneider, C. A., Rasband, W. S. and Eliceiri, K. W. (2012). NIH Image to Image J 25 years of image analysis. Nat. Methods 9, 671-675. doi:10.1038/nmeth.2089

Shrestha, R. L. and Draviam, V. M. (2013). Lateral to end-on conversion of chromosome-microtubule attachment requires kinesins CENP-E and MCAK Curr. Biol. 23, 1514-1526. doi:10.1016/j.cub.2013.06.040

Sikirzhytski, V., Renda, F., Tikhonenko, I., Magidson, V., McEwen, B. F. and Khodjakov, A. (2018). Microtubules assemble near most kinetochores during early prometaphase in human cells. J. Cell Biol. 217, 2647-2659. doi:10.1083/jcb. 201710094

Suijkerbuijk, S. J. E., Vleugel, M., Teixeira, A. and Kops, G. P. J. (2012) Integration of kinase and phosphatase activities by BUBR1 ensures formation of stable kinetochore-microtubule attachments. Dev. Cell 23, 745-755. doi:10.1016/ j.devcel.2012.09.005

Tan, S. (2001). A modular polycistronic expression system for overexpressing protein complexes in Escherichia coli. Protein Expr. Purif. 21, 224-234. doi:10. 1006/prep.2000.1363

Vitre, B., Gudimchuk, N., Borda, R., Kim, Y., Heuser, J. E., Cleveland, D. W. and Grishchuk, E. L. (2014). Kinetochore-microtubule attachment throughout mitosis potentiated by the elongated stalk of the kinetochore kinesin CENP-E. Mol. Biol. Cell 25, 2272-2281. doi:10.1091/mbc.e14-01-0698

Vleugel, M., Hoek, T. A., Tromer, E., Sliedrecht, T., Groenewold, V., Omerzu, M. and Kops, G. J. (2015). Dissecting the roles of human BUB1 in the spindle assembly checkpoint. J. Cell Sci. 128, 2975-2982. doi:10.1242/jcs.169821

Whitmore, L. and Wallace, B.A. (2008) Protein secondary structure analyses from circular dichroism spectroscopy: methods and reference databases. Biopolymers 89, 392-400. doi:10.1002/bip.20853

Wood, K. W., Sakowicz, R., Goldstein, L. S. and Cleveland, D. W. (1997). CENP $E$ is a plus end-directed kinetochore motor required for metaphase chromosome alignment. Cell 91, 357-366. doi:10.1016/S0092-8674(00)80419-5

Yao, X., Anderson, K. L. and Cleveland, D. W. (1997). The microtubule-dependent motor centromere-associated protein $\mathrm{E}$ (CENP-E) is an integral component of kinetochore corona fibers that link centromeres to spindle microtubules. J. Cell Biol. 139, 435-447. doi:10.1083/jcb.139.2.435

Yen, T. J., Compton, D. A., Wise, D., Zinkowski, R. P., Brinkley, B. R., Earnshaw, W. C. and Cleveland, D. W. (1991). CENP-E, a novel human centromereassociated protein required for progression from metaphase to anaphase. EMBO J. 10, 1245-1254. doi:10.1002/j.1460-2075.1991.tb08066.x

Zhang, X. D., Goeres, J., Zhang, H., Yen, T. J., Porter, A. C. and Matunis, M. J. (2008). SUMO-2/3 modification and binding regulate the association of CENP-E with kinetochores and progression through mitosis. Mol. Cell 29, 729-741. doi:10. 1016/j.molcel.2008.01.013

Zhu, M., Wang, F., Yan, F., Yao, P. Y., Du, J., Gao, X., Wang, X., Wu, Q., Ward, T., $\mathbf{L i}$, J. et al. (2008). Septin 7 interacts with centromere-associated protein $E$ and is required for its kinetochore localization. J. Biol. Chem. 283, 18916-18925. doi:10. 1074/jbc.M710591200 


\section{Supplementary Figure 1}

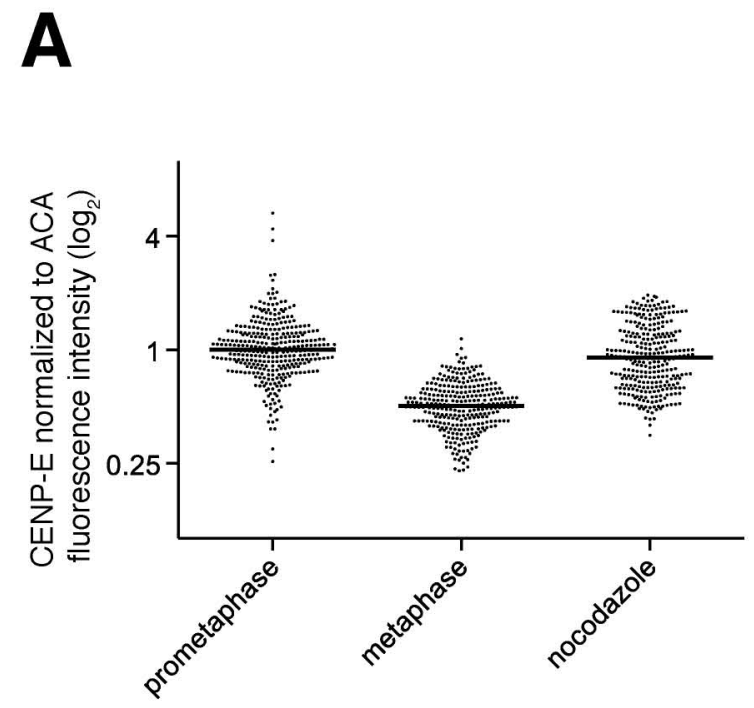

B

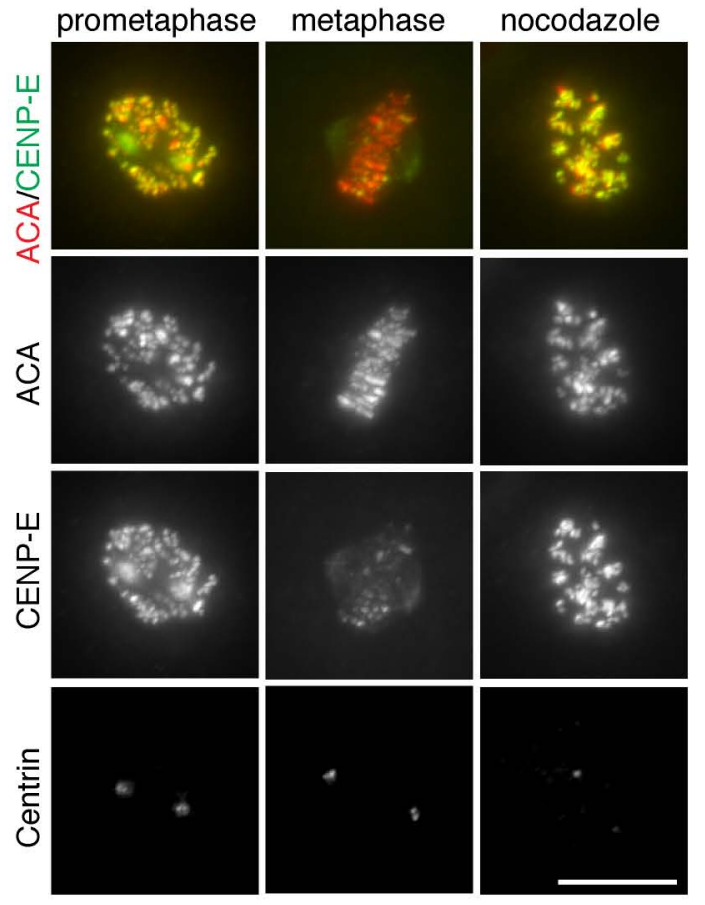

C
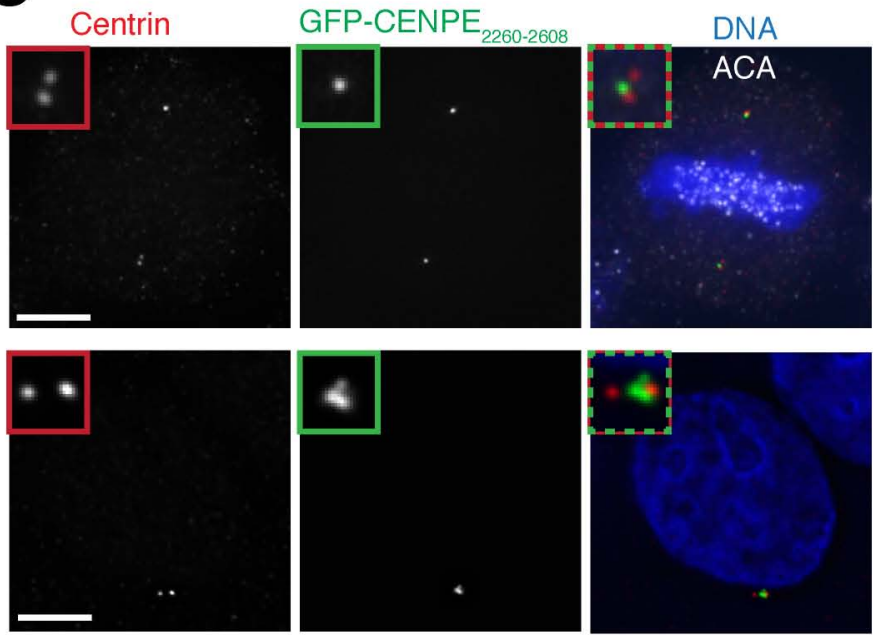

D
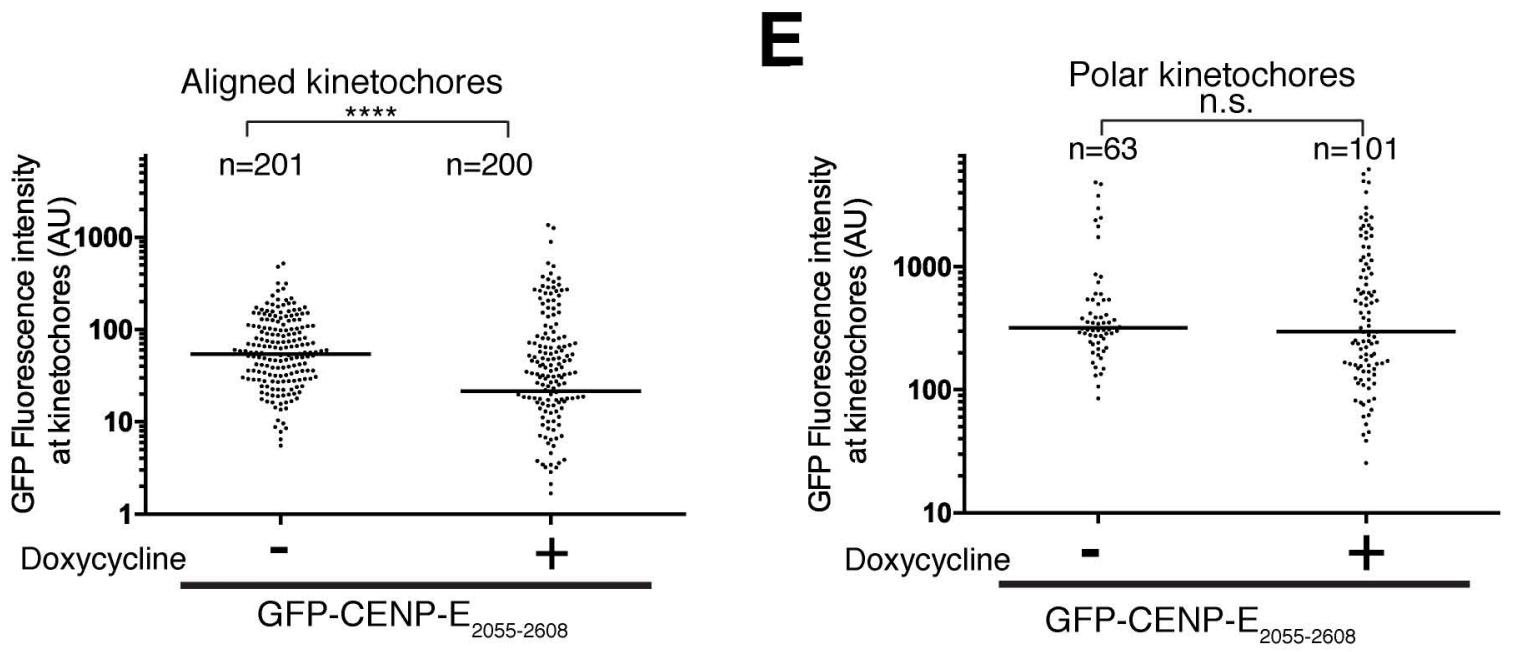
Figure S1: (A) Scatter dot plot showing the quantification of CENP-E intensity normalized to ACA (logarithmic scale) in prometaphase cells, metaphase cells and cells treated with nocodazole. Each point represents the intensity of CENP-E over ACA at one kinetochore. Black line represents the median. (B) Representative immunofluorescence images of HeLa cells for quantification in (A). (C) Representative immunofluorescence images of HeLa cells transfected with GFP-CENP-E ${ }_{2260-2608}$ and stained for kinetochores (ACA), centrioles (centrin2) and DNA (Hoechst). Scale bar: $10 \mu \mathrm{M}$. (D, E) Scatter dot plots (logarithmic scale) showing quantification of GFP-CENP-E $2055-2608$ fluorescence intensity at kinetochores in the presence and absence of endogenous CENP-E (knockdown using inducible CRISPR-Cas9) for non polar kinetochores (D) and polar kinetochores (E). Each point represents the intensity of GFP-CENP- $E_{2055-2608}$ at one kinetochore. Black line represents the median. Asterisks indicate ordinary unpaired T-test significance value. ${ }^{* * * *} \mathrm{P}<0.0001$. Experiments performed three times. 


\section{Supplementary Figure 2}

A
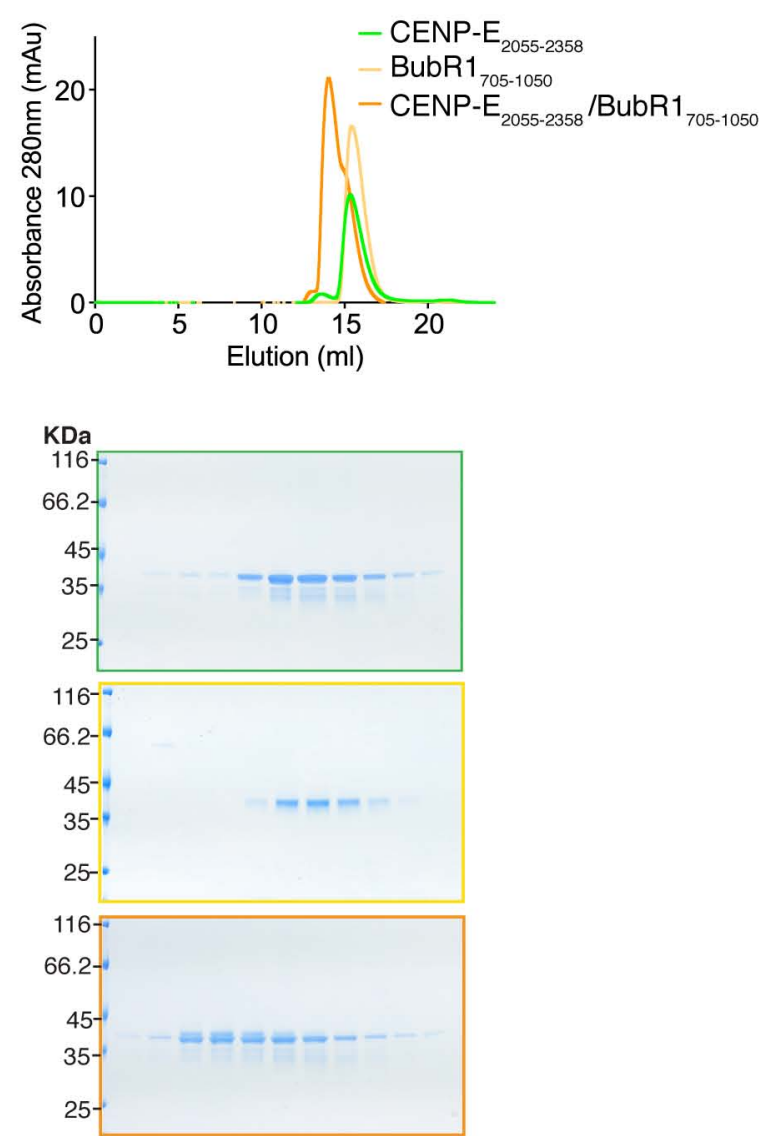

B
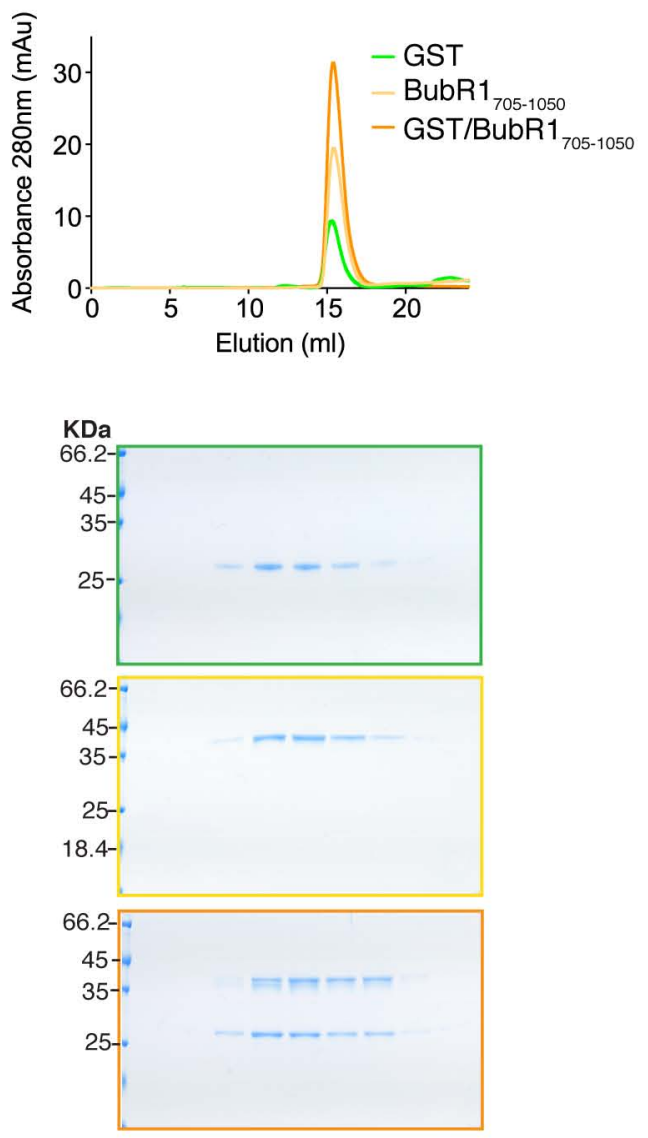

Figure S2: (A) Top, SEC analysis and elution profile for CENP-E E $_{2055-2358}$ (green), BubR1 $705-1050$ (yellow) and CENP-E $2055-2358 / B u b R 1_{705-1050}$ (orange). Bottom, Coomassie-stained gels showing elution profiles for the corresponding protein complexes. (B) Top, SEC analysis and elution profile for GST (green), BubR1 ${ }_{705-1050}$ (yellow) and GST/BubR1 ${ }_{705-1050}$ (orange). Bottom, Coomassie-stained gels showing elution profiles for the corresponding protein complexes, showing GST does not interact with BubR1. 


\section{Supplementary Figure 3}

A

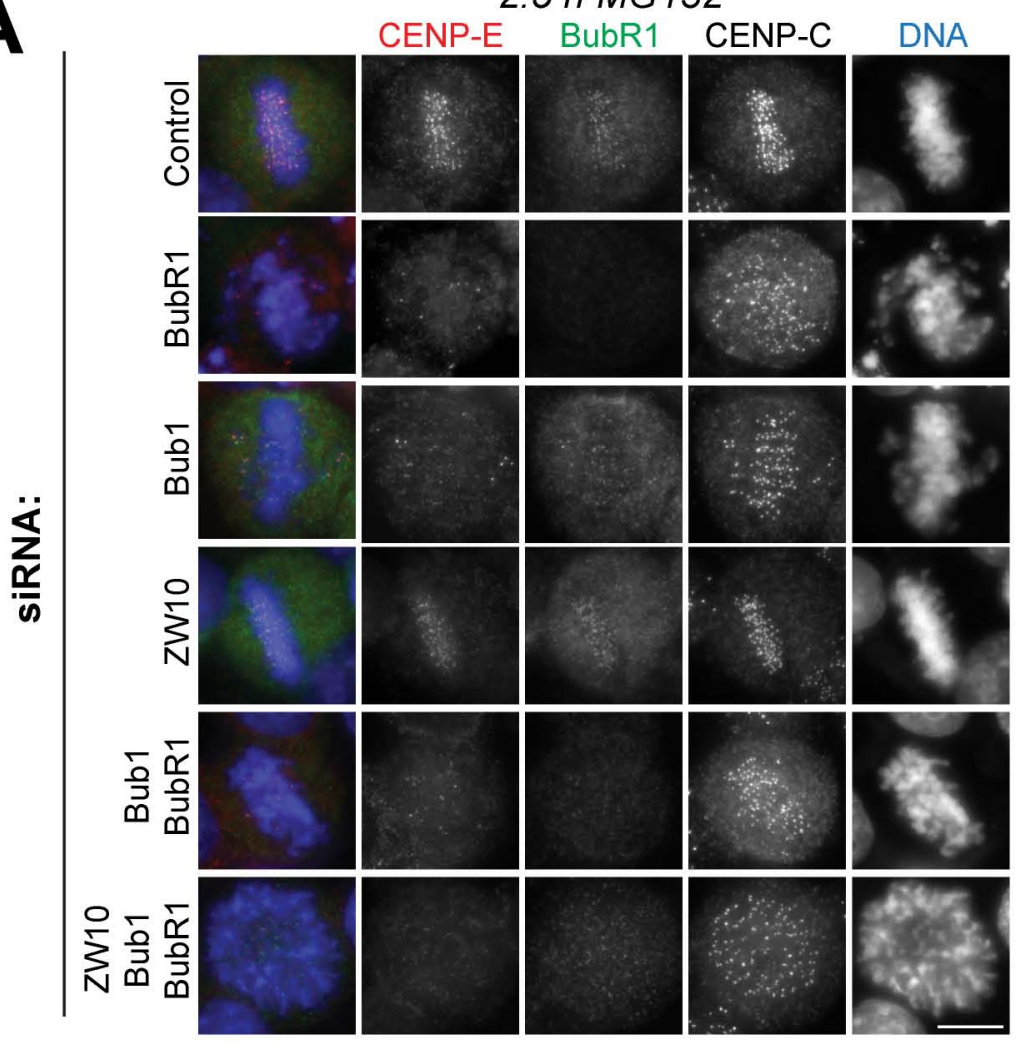

C

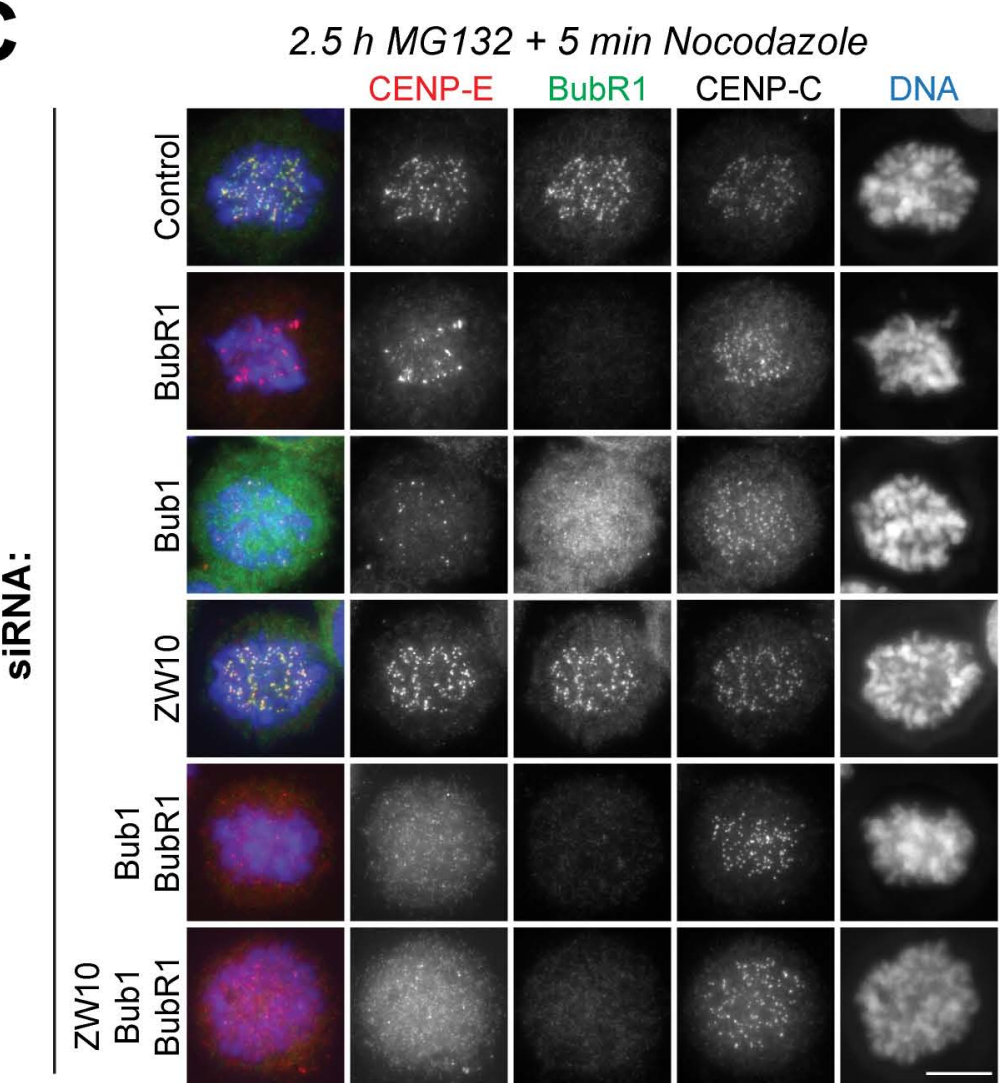

B

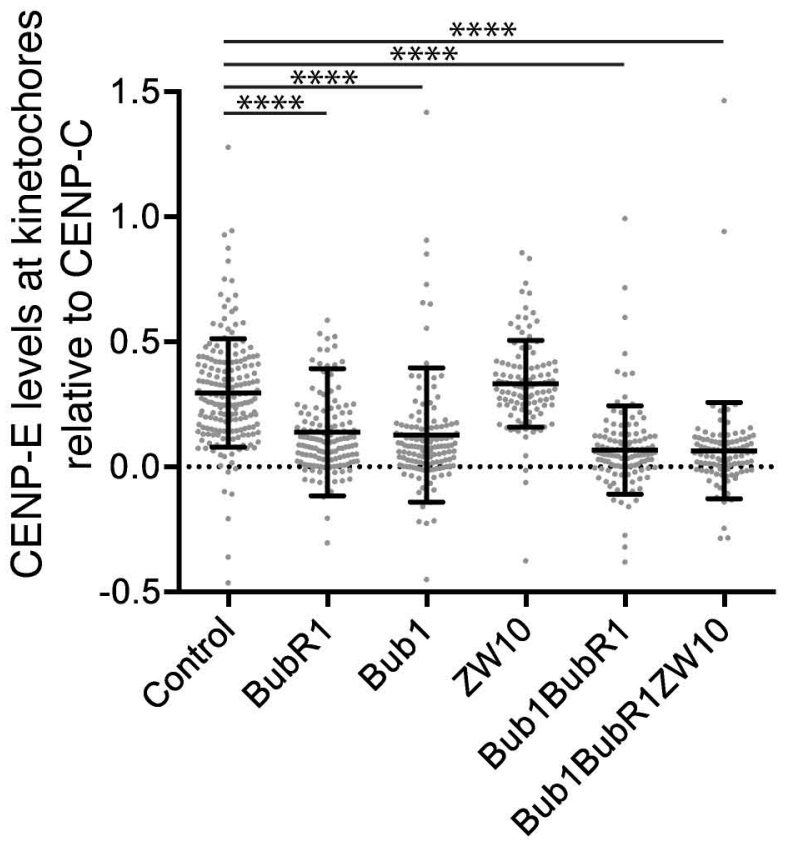

D

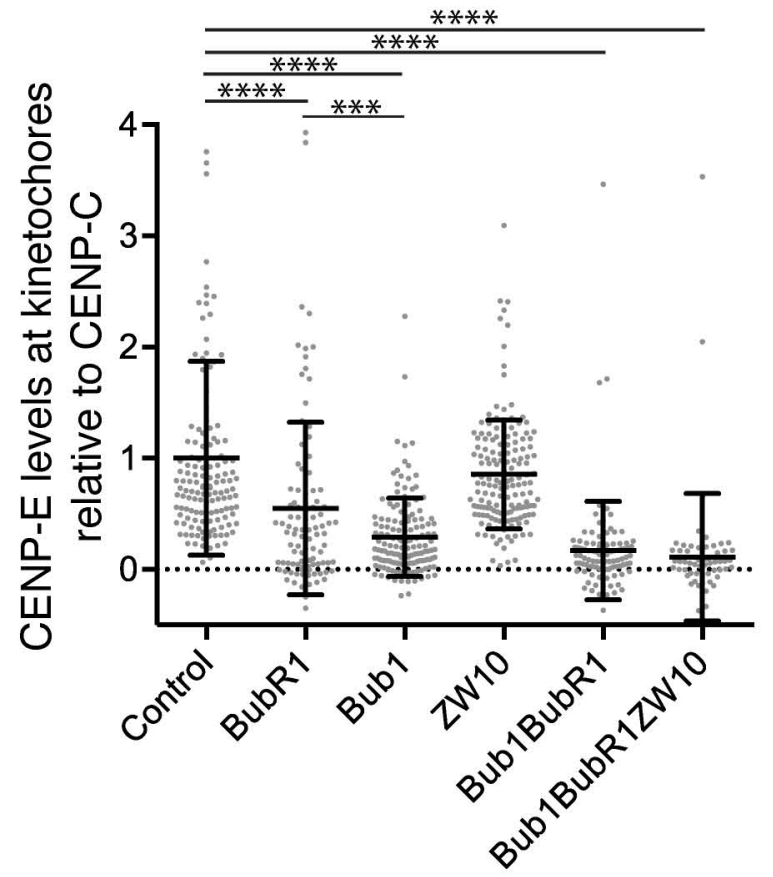


Figure S3: (A, C) Representative immunofluorescence images of HeLa cells treated with indicated siRNA and treated with MG132 for 2.5 hours with and without 5 minutes nocodazole treatment. Cells were stained with CENP-E, BubR1, CENP-C and Hoechst. Scale bar: 10 $\mu \mathrm{m}$. (B, D) Scatter dot plot showing the quantification of CENP-E intensity normalized to CENP-C. Only kinetochores at the metaphase plate were analysed (B). Numbers of kinetochores analyzed for cells treated with $2.5 \mathrm{~h} \mathrm{MG132}$ and $2.5 \mathrm{~h}$ MG132+ 5 minutes nocodazole after RNAi depletion were respectively: $n$ control $=185,140 ; n_{\text {BuBR } 1}=140,98$;

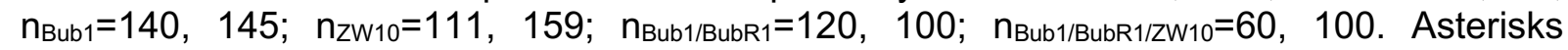
indicate significance value performed using a ANOVA one-way test. ${ }^{* * * *}$ indicate a $\mathrm{P}$ value $<0.0001$. Experiments performed twice.

Table S1: list of proteins found after CENP- $E_{2055-2358}$ pulldown by mass spectrometry

Click here to Download Table S1 